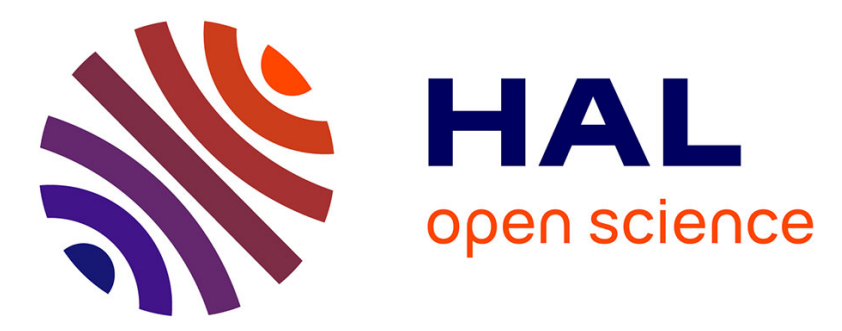

\title{
Closed-loop control of vortex breakdown: A model study
} François Gallaire, Jean-Marc Chomaz, Patrick Huerre

\section{To cite this version:}

François Gallaire, Jean-Marc Chomaz, Patrick Huerre. Closed-loop control of vortex breakdown: A model study. Journal of Fluid Mechanics, 2004, 511 (july), pp.67-93. 10.1017/s0022112004009498 . hal-01024951

\section{HAL Id: hal-01024951 \\ https://hal-polytechnique.archives-ouvertes.fr/hal-01024951}

Submitted on 29 Jul 2014

HAL is a multi-disciplinary open access archive for the deposit and dissemination of scientific research documents, whether they are published or not. The documents may come from teaching and research institutions in France or abroad, or from public or private research centers.
L'archive ouverte pluridisciplinaire HAL, est destinée au dépôt et à la diffusion de documents scientifiques de niveau recherche, publiés ou non, émanant des établissements d'enseignement et de recherche français ou étrangers, des laboratoires publics ou privés. 


\title{
Closed-loop control of vortex breakdown: a model study
}

\author{
By FRANÇOIS GALLAIRE†, JEAN-MARC CHOMAZ \\ AND PATRICK HUERRE \\ Laboratoire d'Hydrodynamique (LadHyX), CNRS-Ecole Polytechnique, \\ F-91128 Palaiseau, France
}

(Received 31 May 2002 and in revised form 16 January 2004)

The objective of the study is to develop a closed-loop control scheme that is capable of preserving the columnar swirling flow state in the finite-length pipe model of Wang \& Rusak (1996a). The base state consists of a solid body rotation superimposed on axial plug flow, with two dimensionless parameters: the swirl $\Omega$ and the pipe aspect ratio $L$. The linear stability properties of the columnar base state are documented and shown to give rise to unstable global modes above a critical swirl level $\Omega_{1}$, thereby triggering vortex breakdown. Our study focuses on the derivation of a control method in order to quench the linear development of the Wang \& Rusak instability. An optimal control approach is then devised for a reduced-order system which is obtained by a suitable projection on a low-order subspace of the $N$ least-stable eigenmodes. The actuator consists of perturbations of the inlet circulation and its time history is selected so as to minimize a cost-functional incorporating both the state energy and the control energy. A Riccati-based formulation leads to the determination of the optimal gain matrix for the low-order system. When applied to the full linear system, the feedback law for $N=4$ succeeds in maintaining the columnar base state for swirl levels as high as $13 \%$ above global onset. The control scheme is found to be robust with respect to noise and to uncertainties in parameter settings. It remains effective even under partial-state information conditions.

\section{Introduction}

Vortex breakdown is a widespread phenomenon that affects a variety of flow situations, from leading-edge vortices on delta wings to atmospheric tornadoes and flame holders in combustion devices. It consists of a sudden change in the topology of the streamlines signalled by the appearance of a stagnation point within the flow. From a more fundamental point of view, it is best analysed on generic configurations such as a free vortex with axial flow, rotating flow in pipes, or in closed cylindrical containers. The objective of the present study is to design an optimal closed-loop flow control scheme which prevents vortex breakdown in the idealized setting of a columnar vortex with axial flow contained in a circular pipe of finite length.

Vortex breakdown was first observed experimentally by Peckham \& Atkinson (1957) on gothic wings at sufficiently high angle of attack. Later experimental studies (Elle 1958; Werlé 1960; Lambourne \& Bryer 1961) have focused on the delta wing

$\dagger$ Present address: Laboratorie J.-A. Dieudonné, Université de Nice, Sophia Antipolis - CNRS, Parc Valrose, F 06108 Nice Cedex 02, France. 
geometry and identified two main types of vortex breakdown: axisymmetric bubbles and spirals. It was soon recognized that, independently of the particular geometry under consideration, the key control parameter is the relative magnitude of the swirl and the axial flow, namely the swirl parameter. This observation has led to the examination of vortex breakdown in idealized configurations where the swirl appears as an explicit parameter. In this context, swirling flow in a circular pipe has been of primary interest. Since a theoretical model of this flow is being considered in the present study, we briefly summarize previous experimental and numerical investigations pertaining to this geometry only. Harvey (1962) appears to have been the first to make detailed observations and to measure the amount of swirl necessary to trigger vortex breakdown. Sarpkaya $(1971,1974)$ identified three kinds of vortex breakdown, the bubble, the spiral and the double helix, and emphasized the important role of the applied axial pressure gradient. Several other breakdown states were detected and analysed quantitatively by Faler \& Leibovich $(1977 a, b)$ and Escudier, Bornstein \& Maxworthy (1982) among others. More recently, Brücker \& Althaus (1992), Brücker (1993) and Brücker \& Althaus (1995) have thoroughly documented experimentally the spatio-temporal dynamics of breakdown in confined tubes: the topology of the flow within the tube was shown to be dominated by the existence of a precessing inclined vortex ring. For a review of vortex breakdown observations in pipes and closed geometry containers, the reader is referred to Leibovich (1978, 1984), Escudier (1987) or Althaus, Brücker \& Weimer (1996).

The first numerical axisymmetric study of vortex breakdown in pipes was conducted by Kopecky \& Torrance (1973). Beran \& Culick (1992) demonstrated via a numerical search of stationary solutions that the onset of breakdown is subcritical, i.e. there exists an hysteresis loop where both the columnar and vortex breakdown states coexist. The unsteady direct numerical simulations of Brown \& Lopez (1990), Lopez (1994) and Darmofal (1996) confirmed the subcritical nature of the bifurcation and interpreted breakdown as the end result of the tilting of axial vorticity into its azimuthal counterpart.

Over the last forty years, numerous theories and models of vortex breakdown have been proposed and the reader is referred to the articles of Hall (1972), Leibovich (1978, 1984), Escudier (1987), Delery (1994) and Shtern \& Hussain (1996) for comprehensive reviews. We focus here solely on theoretical aspects that are directly relevant to our analysis. Within an inviscid and axisymmetric assumption, Squire (1960) established a criterion for vortex breakdown, corresponding to the critical amount of swirl necessary to sustain a stationary wave of infinite wavelength. In the bulk of the present paper, this swirl value corresponds to $\Omega_{B}$. According to Benjamin (1962), vortex breakdown emerges as a transition of swirling flows from supercritical to subcritical. A columnar swirling flow is said to be supercritical if it can only support downstream travelling Kelvin waves in the low-wavenumber limit. Conversely, it is said to be subcritical if it can support both upstream and downstream travelling Kelvin waves. However, the mechanisms by which this transition is induced remained unspecified. The swirl value separating supercritical and subcritical columnar states precisely coincides with the value $\Omega_{B}$ obtained by Squire (1960). It has in fact become customary to ascribe the subscript $B$ to this particular swirl setting.

Wang \& Rusak (1997a) chose instead to consider the nonlinear states sustained by a vortex with axial flow in a pipe of finite length, for specific upstream and downstream boundary conditions. A functional is introduced, the extrema of which correspond to steady nonlinear states. The following bifurcation sequence (see sketch in figure 2) is then identified as the swirl parameter is increased. A first saddle-node 
bifurcation is encountered at $\Omega=\Omega_{0}$ which gives rise to a stable breakdown state and an unstable solitary wave state, while the basic columnar state remains stable. Above a higher critical swirl value $\Omega_{1}$, the columnar vortex loses its stability in favour of the accelerated state, as demonstrated by Wang \& Rusak (1996a, $b$ ), while the breakdown state continues to be a stable state, corresponding to the global minimum of the functional. This loss of stability occurs slightly above the swirl value $\Omega_{B}$ of Squire (1960) at which stationary waves of infinite wavelength are sustained and upstream propagation becomes possible, the difference arising from finite-length effects. It should be emphasized that the instability of the columnar state is global in nature: the bulk of the vortex remains locally neutrally stable. The stability analysis is said to be local when the flow is assumed to be infinite in the axial direction and can be therefore decomposed into Fourier components of wavenumber $k$. The flow is further referred to as neutral when the dispersion relation yields purely imaginary eigenvalues only. In the present case, the bulk of the vortex therefore only acts as a waveguide for travelling Kelvin waves. The upstream and downstream boundaries constitute the destabilizing agent leading to global onset. There is no need for a local instability mechanism in the bulk of the pipe, i.e. vortex flows can be stable according to classical stability criteria (Rayleigh 1916; Howard \& Gupta 1962) but unstable according to the Wang \& Rusak mechanism. This theory provides a consistent explanation of the axisymmetric vortex breakdown process. Its extension to weak viscosity (Wang \& Rusak 1997b) is in good qualitative agreement with the numerical Navier-Stokes simulations of Beran \& Culick (1992), Lopez (1994) and Beran (1994).

Previous vortex breakdown control studies have all been experimental and have dealt with the technologically relevant delta wing configuration, where the appearance of vortex breakdown is known to alter the manoeuvrability of the wing. The great majority have focused on open-loop control design. The first successful attempt appears to have been carried out by Werlé (1960) who demonstrated that suction downstream of the nominal breakdown point succeeded in postponing it further downstream. More recently, Vorobieff \& Rockwell (1996) have shown that intermittent blowing at the trailing edge was also effective in delaying vortex breakdown. Gursul, Srinivas \& Batta (1995) appear to be the only investigators to have successfully implemented a closed-loop control scheme based on piezo-electric pressure probes as sensors and varying sweep angle as actuator. The feedback loop was designed to maintain the position of vortex breakdown under changes in angle of attack. An excellent review of experimental control strategies is given by Mitchell \& Delery (2001).

To our knowledge, there are no previous studies of open- or closed-loop control in the simpler pipe geometry. The present study relies, in large measure, on recent implementations of optimal control theory to unstable bounded shear flows such as boundary layers and plane channel flow. A general review of these developments is given in Bewley (2001). In accordance with the approach taken here, we only mention flow control studies based on a preliminary discretization of the equations of fluid motion, so as to be able to directly apply the techniques of optimal control to a discrete dynamical system ultimately cast in the standard state-space form $\dot{\boldsymbol{x}}=\boldsymbol{A} \boldsymbol{x}+\boldsymbol{B u}$.

Following the proportional-integral-differential (PID) control approach developed by Joshi, Speyer \& Kim (1997), Cortelezzi \& Speyer (1998) and Bewley \& Liu (1998) applied optimal and robust control theory to the stabilization of plane channel flow by suction and blowing at the walls. The formulation consisted in suitably discretizing the linear Orr-Sommerfeld-Squire equations for a single Fourier mode on a finite number of collocation points in order to end up with a discrete dynamical system. The optimal control gain matrix in the feedback loop is then obtained by solving an algebraic 
Riccati equation, in order to minimize an appropriate cost-functional measuring the intensity of the flow disturbances and of the applied control. The formulation was shown by Bewley \& Liu (1998) to effectively preserve plane Poiseuille flow both supercritically, when the flow is linearly unstable, and subcritically, when it may exhibit long-lived transients. In the same flow geometry, Cortelezzi \& Speyer (1998) resorted to a similar a priori discretization and focused instead on the preliminary identification of a suitable subset of controllable two-dimensional modes. A reduced low-order model was derived in the subspace of these modes. An optimal and robust control scheme was then computed for the reduced-order system which, when applied to two-dimensional simulations of plane channel flow, led to a $90 \%$ wallshear stress reduction. This strategy was further extended to reduce the wall-shear stress disturbances in a turbulent three-dimensional channel flow by Cortelezzi et al. (1998) and Lee et al. (2001). Högberg, Bewley \& Henningson (2003) have recently demonstrated that the gains calculated by Bewley \& Liu (1998), after a suitable inverse Fourier transform into physical space, led to spatially localized convolution kernels that are optimal for controlling three-dimensional perturbations. More strikingly, these kernels result in the successful quenching of transition in direct numerical simulations of the full nonlinear equations of fluid motion. This result provides a strong justification for conducting optimal control studies within a linearized setting as a first step.

The objective of the present analysis is to control the evolution of linearly unstable global modes in the vortex tube model of Wang \& Rusak (1996a, 1997a). The actuator consists of perturbations of the inlet circulation that are selected so as to minimize a suitably defined cost-functional incorporating the intensity of both the flow disturbances and the applied perturbations. In other words, we wish to vary the inlet circulation with time so as to restore the stability of the columnar flow beyond the critical value $\Omega_{1}$ for global instability (see sketch in figure 2). Such an actuator may be easily implemented experimentally through a variation of the swirl generator rotation rate as in Billant, Chomaz \& Huerre's (1998) experiment, or of the vane angle as in Harvey (1962) or Sarpkaya (1971). It is thereby expected that such inlet forcing will prevent the full nonlinear system from jumping into the vortex breakdown state, as long as infinitesimal perturbations are injected in the flow.

The formulation proceeds in four distinct steps. First a linear global instability analysis is performed for a finite-length columnar base state consisting of plug axial flow and solid body rotation. This step extends Wang \& Rusak's (1996a) global instability study to all values of the swirl $\Omega$. A reduced-order discrete dynamical system is subsequently derived by projection onto a subset of least stable modes. Optimal full-state information control theory is then implemented to determine the optimal control gain for this reduced system. In a final step, this gain control law is implemented on the full linear system.

The study is organized according to the following outline: the general formulation and the main results of Wang \& Rusak's analysis are presented and summarized in $\S 2$. The global linear instability analysis is performed in $\S 3$. In $\S 4$, the discrete reducedorder system is derived and a corresponding optimal feedback law is determined. Its effectiveness for the full system is evaluated in $\S 5$. The presentation continues with a parametric study and an assessment of various robustness issues in $\S 6$. Section 7 is devoted to issues associated with the practical implementation of the above control strategy. Different choices of actuators are discussed in terms of their effectiveness in delaying vortex breakdown onset. Furthermore, we account for the fact that, in any real situation, only partial information on the state of the system is available 


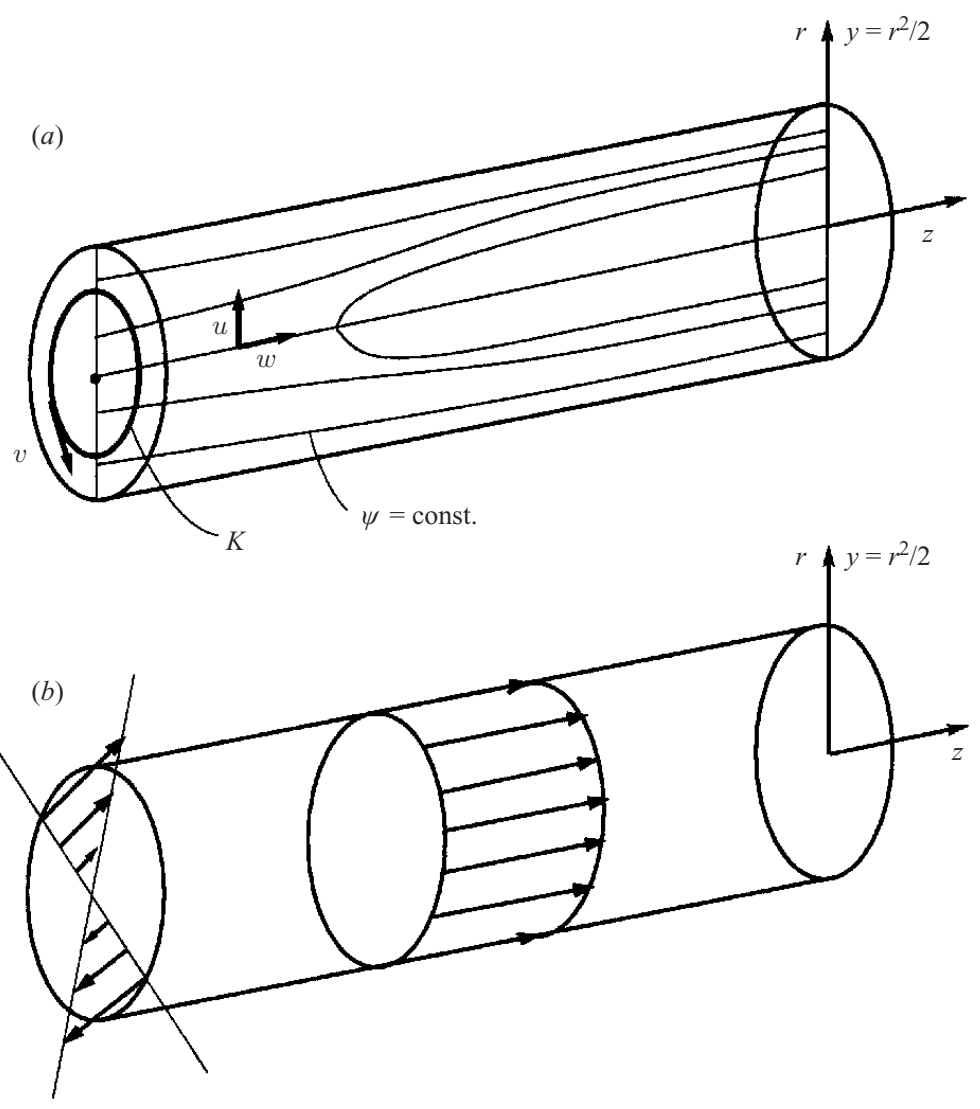

FIGURE 1. Flow configuration. (a) Sketch of breakdown state and essential flow variables; axisymmetric streamsurfaces $\psi(y, z, t)=$ const. have been represented by their intersections with a meridional plane. $(b)$ Base flow consisting of plug axial flow and solid body rotation.

at discrete sensor locations. In concluding remarks, we summarize our findings and discuss some of the limitations of the present approach as well as future developments.

\section{General formulation}

\subsection{Squire-Long equations}

Consider axisymmetric swirling flow in a pipe of radius $R$ and length $l$. The viscosity is set to zero so that the flow is governed by the Euler equations. We use cylindrical coordinates $(r, \theta, z)$ and the velocity components $(u, v, w)$ correspond respectively to the radial, azimuthal and axial velocity. The flow variables used in the study are sketched in figure $1(a)$.

The base flow sketched in figure $1(b)$ consists of an axial flow $w_{0}$ superimposed on a uniform rotation around the $z$-axis of constant axial vorticity $2 \omega$. Throughout the paper, we adopt $R$ as length scale and $w_{0}$ as velocity scale, so that the dimensionless columnar base flow is

$$
u=0, \quad v=\sqrt{\Omega} r / 2, \quad w=1,
$$

for $0 \leqslant r \leqslant 1,0 \leqslant z \leqslant L$, with $L$ being the non-dimensional pipe length $L=l / R$ and $\Omega$ being the swirl parameter $\Omega=4 \omega^{2} R^{2} / w_{0}^{2}$. The model problem considered in the 
present investigation is seen to involve two control parameters: the swirl $\Omega$ and the pipe aspect ratio $L$. In what follows, the non-dimensional pipe length is set to $L=10$ and only $\Omega$ is varied. The influence of the parameter $L$ on the stability properties is discussed in Wang \& Rusak (1996a). At a given swirl above threshold, the number of unstable modes increases with $L$.

Since the flow is axisymmetric, it is governed by the so-called unsteady Squire-Long equations (see Szeri \& Holmes 1988, and references therein)

$$
\begin{gathered}
K_{t}+\psi_{y} K_{z}-\psi_{z} K_{y}=0, \\
\chi_{t}+\psi_{y} \chi_{z}-\psi_{z} \chi_{y}=\frac{1}{4 y^{2}}\left(K^{2}\right)_{z}, \\
\chi=-\left(\psi_{y y}+\psi_{z z} / 2 y\right) .
\end{gathered}
$$

In the above system $K(r, z, t)=r v$ is the azimuthal circulation, $\psi(r, z, t)$ is the stream-function such that $u=-\psi_{z} / r$ and $w=\psi_{r} / r$ and $y=r^{2} / 2$ is a rescaled radial coordinate. Instead of working with the azimuthal vorticity $\eta$, the reduced vorticity $\chi=\eta / r$ has been introduced. In terms of the flow variables $K, \psi$ and $\chi$, the columnar base flow defined in (2.1) takes the form

$$
K_{0}(y)=\sqrt{\Omega} y, \quad \psi_{0}(y)=y, \quad \chi_{0}(y)=0 .
$$

Note that, according to (2.2), the azimuthal circulation $K(y, z, t)$ is a conserved quantity which is simply transported along the flow (an expression of Kelvin's theorem) whereas the azimuthal vorticity evolution equation (2.3) accounts for the tilting of the vorticity via its right-hand side. The stretching of the azimuthal vorticity is implicitly included through the use of the reduced azimuthal vorticity $\chi=\eta / r$. As discussed in Szeri \& Holmes (1988), the problem is well-posed once an inlet condition for $K$ and $\chi$ and a boundary condition for $\psi$ on the pipe surface and at the ends are specified. Since $y=0$ and $y=1 / 2$ are streamsurfaces, the lateral boundary conditions are

$$
\begin{gathered}
\psi(0, z, t)=0, \\
\psi(1 / 2, z, t)=1 / 2 .
\end{gathered}
$$

Condition (2.6) results from axisymmetry whereas condition (2.7) sets the total mass flux through the pipe. The following three inlet conditions are added:

$$
\begin{aligned}
\psi(y, 0, t) & =\psi_{0}(y), \\
K(y, 0, t) & =K_{0}(y), \\
\chi(y, 0, t) & =\chi_{0}(y) .
\end{aligned}
$$

These conditions effectively prescribe that the base flow be maintained at the inlet $z=0$ in the presence of perturbations, allowing the inlet state a degree of freedom to develop a radial velocity however. The outlet condition is the same as in Wang \& Rusak (1996a,b, 1997a):

$$
\psi_{z}(y, L, t)=0 .
$$

A vanishing radial velocity is thereby imposed at the outlet. Note that the columnar base flow, $K_{0}(y), \psi_{0}(y)$ and $\chi_{0}(y)$ is an exact solution of the inviscid nonlinear equations (2.2)-(2.11).

\subsection{Wang \& Rusak's analysis}

Wang \& Rusak (1997a) have analysed in detail the sequence of stationary states $(\partial / \partial t=0)$ of the Squire-Long equations $(2.2)-(2.11)$ as a function of the swirl 


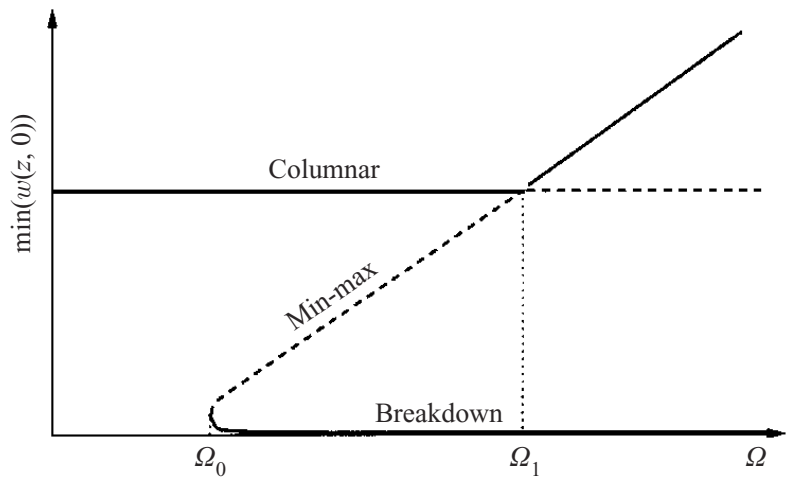

FIGURE 2. Qualitative bifurcation diagram of flow states as a function of swirl parameter $\Omega$ in Wang \& Rusak's analysis. The state is described by its minimum axial velocity on the axis.

parameter $\Omega=4 \omega^{2} R^{2} / w_{o}^{2}$. The qualitative results of their study, as sketched in figure 2, apply not only to the base flow (2.5), but also to general entrance profiles which are more representative of experimental situations.

Below the value $\Omega_{0}=14.68$, the only possible state is the stable columnar vortex. As $\Omega$ increases above $\Omega_{0}$, a saddle-node bifurcation takes place, giving rise to a stable breakdown state and an unstable accelerated state. At the second, higher critical value $\Omega_{1}=14.70$, the columnar state loses its stability through a transcritical bifurcation in favour of the accelerated state. In the case of base flow (2.5), Keller, Egli \& Exley (1985) and Wang \& Rusak (1997a) have shown that $\Omega_{1}=\Omega_{0}+\pi^{2} / 4 L^{2}$ : at sufficiently large aspect ratios, the bifurcation diagram in figure 2 is only mildly subcritical. Base flow (2.5) is indeed somewhat unusual: the critical swirl $\Omega_{B}$, as defined in the introduction, equals $\Omega_{0}$.

\section{Linear instability analysis}

\subsection{Linearized equations}

Following Wang \& Rusak (1996a), let us superimpose the perturbations, indicated by primed variables:

$$
\begin{aligned}
& K(y, z, t)=K_{0}(y)+K^{\prime}(y, z, t), \\
& \psi(y, z, t)=\psi_{0}(y)+\psi^{\prime}(y, z, t), \\
& \chi(y, z, t)=\chi^{\prime}(y, z, t),
\end{aligned}
$$

and linearize the Squire-Long equations (2.2)-(2.11) around the columnar base flow. It is convenient to introduce the basis of radial functions $f_{i}(y)=\sqrt{y} J_{1}\left(b_{1 i} \sqrt{2 y}\right)$ for $i=1 \ldots \infty$, where $J_{1}$ is the Bessel function of order unity and $b_{1 i}$ its $i$ th zero. These functions satisfy the radial boundary conditions for the perturbations, $f_{i}(0)=$ $f_{i}(1 / 2)=0$. The perturbations are then expanded on this basis, according to

$$
\begin{aligned}
K^{\prime}(y, z, t) & =\sum_{i=1}^{\infty} K^{(i)}(z, t) f_{i}(y), \\
\psi^{\prime}(y, z, t) & =\sum_{i=1}^{\infty} \psi^{(i)}(z, t) f_{i}(y) .
\end{aligned}
$$


Substituting the above expansions into the linearized equations, lead to the infinite number of uncoupled one-dimensional partial differential equations:

$$
\left[\begin{array}{cc}
1 & 0 \\
0 & b_{1 i}^{2}-\frac{\partial^{2}}{\partial z^{2}}
\end{array}\right]\left(\begin{array}{c}
\dot{K}^{(i)} \\
\dot{\psi}^{(i)}
\end{array}\right)=\left[\begin{array}{cc}
-\frac{\partial}{\partial z} & \sqrt{\Omega} \frac{\partial}{\partial z} \\
\sqrt{\Omega} \frac{\partial}{\partial z} & \frac{\partial^{3}}{\partial z^{3}}-b_{1 i}^{2} \frac{\partial}{\partial z}
\end{array}\right]\left(\begin{array}{l}
K^{(i)} \\
\psi^{(i)}
\end{array}\right),
$$

with the boundary conditions

$$
K^{(i)}(0, t)=0, \quad \psi^{(i)}(0, t)=0, \quad \psi_{z z}^{(i)}(0, t)=0, \quad \psi_{z}^{(i)}(L, t)=0 .
$$

The dot denotes the time-derivative. The values $\Omega=b_{1 i}^{2}$ are precisely the critical swirls defined by Benjamin (1962). They correspond to the swirl settings above which the $i$ th branch of Kelvin waves in the pipe $0 \leqslant y \leqslant 1 / 2$ admits a stationary solution of zero frequency and finite real axial wavenumber. In particular, the value $\Omega_{B} \equiv b_{11}^{2}$ is the critical swirl first introduced by Squire (1960). Wang \& Rusak (1996a) studied equations (3.2)-(3.3) for the first branch $i=1$ and demonstrated that the columnar base state was stable if and only if $\Omega<\Omega_{1}=\Omega_{B}+\pi^{2} / 4 L^{2}$. For each particular $i$, a similar approach may be followed step by step to demonstrate that equations (3.2)(3.3) lead to stable solutions if and only if $\Omega<b_{1 i}^{2}+\pi^{2} / 4 L^{2}$. Since $b_{1 i+1}>b_{1 i}$ with $b_{11}^{2}=14.68, b_{12}^{2}=49.21$, etc., each branch successively loses its stability for higher and higher swirl values. In the present study, attention is restricted to swirl values in the range $0<\Omega<b_{12}^{2}$, where at most only the first branch is unstable. Higher-order branches remain damped.

\subsection{Global instability}

Global eigenvalues and eigenfunctions of system (3.2)-(3.3) with $i=1$ are now sought in terms of a linear combination of Kelvin waves supported by the columnar base state. Consider Kelvin waves of the form

$$
\begin{aligned}
& K^{(1)}(z, t)=\operatorname{Re}\left\{A \mathrm{e}^{\mathrm{i} k z+\lambda t}\right\}, \\
& \psi^{(1)}(z, t)=\operatorname{Re}\left\{B \mathrm{e}^{\mathrm{i} k z+\lambda t}\right\},
\end{aligned}
$$

where $\operatorname{Re}\{\}$ is the real part of \{\}$, A$ and $B$ are two complex amplitudes, $k$ is the axial wavenumber and $\lambda$ is at this stage an unknown complex eigenvalue. Upon substituting (3.4) into the governing equation (3.2), one readily finds that non-trivial solutions are possible if and only if $k$ and $\lambda$ satisfy the local dispersion relation

$$
\left(k^{2}+\Omega_{B}\right)(\lambda+\mathrm{i} k)^{2}+\Omega k^{2}=0 .
$$

This relation is identical to the one derived by Kelvin (1880) and Chandrasekhar (1961), provided $\lambda$ is replaced by its Doppler-shifted counterpart $\lambda+\mathrm{i} k$ due to the basic advection. For a given $\lambda$, there exist in general four complex spatial branches $k_{1}(\lambda), k_{2}(\lambda), k_{3}(\lambda)$ and $k_{4}(\lambda)$. Imposing the boundary conditions (3.3) on a linear combination of these four waves requires that the eigenvalue $\lambda$ satisfy the global selection relation

$$
D(\lambda)=\left|\begin{array}{cccc}
1 & 1 & 1 & 1 \\
\frac{\sqrt{\Omega} \mathrm{i} k_{1}}{\lambda+\mathrm{i} k_{1}} & \frac{\sqrt{\Omega} \mathrm{i} k_{2}}{\lambda+\mathrm{i} k_{2}} & \frac{\sqrt{\Omega} \mathrm{i} k_{3}}{\lambda+\mathrm{i} k_{3}} & \frac{\sqrt{\Omega} \mathrm{i} k_{4}}{\lambda+\mathrm{i} k_{4}} \\
k_{1}^{2} & k_{2}^{2} & k_{3}^{2} & k_{4}^{2} \\
k_{1} \mathrm{e}^{\mathrm{i} k_{1} L} & k_{2} \mathrm{e}^{\mathrm{i} k_{2} L} & k_{3} \mathrm{e}^{\mathrm{i} k_{3} L} & k_{4} \mathrm{e}^{\mathrm{i} k_{4} L}
\end{array}\right|=0 .
$$




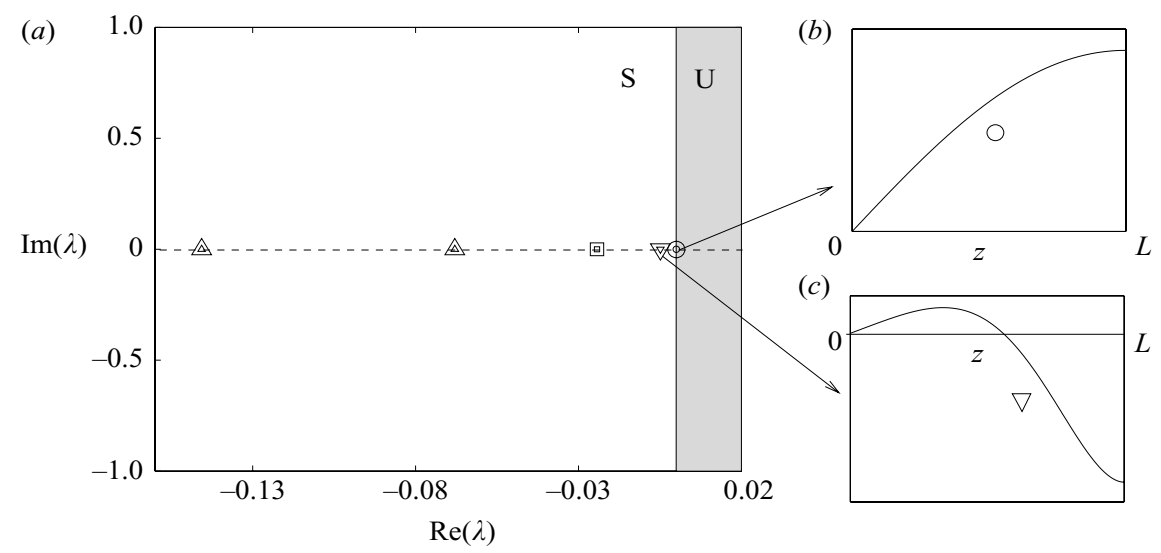

FIGURE 3. Global eigenvalue spectrum and associated eigenmodes at the swirl parameter $\Omega=\Omega_{B}+0.028$. (a) Eigenvalues $\lambda_{i}$ in complex $\lambda$-plane; large symbols: zeros of $D(\lambda)$ given in (3.6); small symbols: corresponding eigenvalues of the discretized equations (5.1); note the presence of only one unstable eigenvalue $\lambda_{1} .(b-c)$ Leading eigenmodes $K_{1}(z)$ and $K_{2}(z)$.

The global spectrum associated with (3.6) is conveniently represented in the complex $\lambda$-plane in figure 3 , at the swirl parameter setting $\Omega=\Omega_{B}+0.028>\Omega_{1}$ (see figure 2), just above the instability threshold of the columnar state. There is only one unstable eigenvalue $\lambda_{1}=2.77 \times 10^{-5}$, all others being negative real. Such a behaviour is the signature of a 'static' instability, characterized by $\omega_{r}=\lambda_{i}=0$, as in the divergence of a buckling beam. To each eigenvalue $\lambda_{i}$ is associated an eigenmode $\boldsymbol{X}_{i}(z)=\left(K_{i}(z), \psi_{i}(z)\right)^{T}$. The first two circulation components $K_{1}(z)$ and $K_{2}(z)$ are also plotted in figure 3. As expected, the number of zeros of $K_{i}(z)$ increases with the mode index $i$. The real parts $\operatorname{Re}(\lambda)$ of the eigenvalues are plotted in figure 4 as a function of the swirl parameter $\Omega-\Omega_{B}$. Note the presence of more and more unstable modes as $\Omega$ is increased. Purely real eigenvalues are indicated by continuous solid curves whereas pairs of complex-conjugate eigenvalues are denoted by dashed lines. The latter curves emerge through the coalescence of purely real eigenvalues, the first one taking place at $\Omega=\Omega_{B}+0.25$. Finally, note that linear instability sets in at a swirl value $\Omega_{1}=\Omega_{B}+0.02$ which is virtually indistinguishable from $\Omega_{B}$ in figure 4 .

\section{Reduced-order optimal control scheme}

The next three sections are devoted to the design of a full-information controller with the inlet circulation as actuator. Considerations on partial-information control and other types of possible actuators are postponed to $\S 7$.

\subsection{Control theory formulation}

One of the first steps in the design of a closed-loop control is to define an actuator (see e.g. Gunzburger 1995). We choose here to act on the inlet circulation through the boundary condition $K^{(1)}(0, t) \equiv U(t)$ which replaces $K^{(1)}(0, t)=0$ in the unforced system (3.3). The function $U(t)$ denotes the unknown control. As in Joshi et al. (1997) we make the change of variables

$$
K^{(1)}(z, t)=K(z, t)+U(t), \quad \psi^{(1)}(z, t)=\psi(z, t),
$$




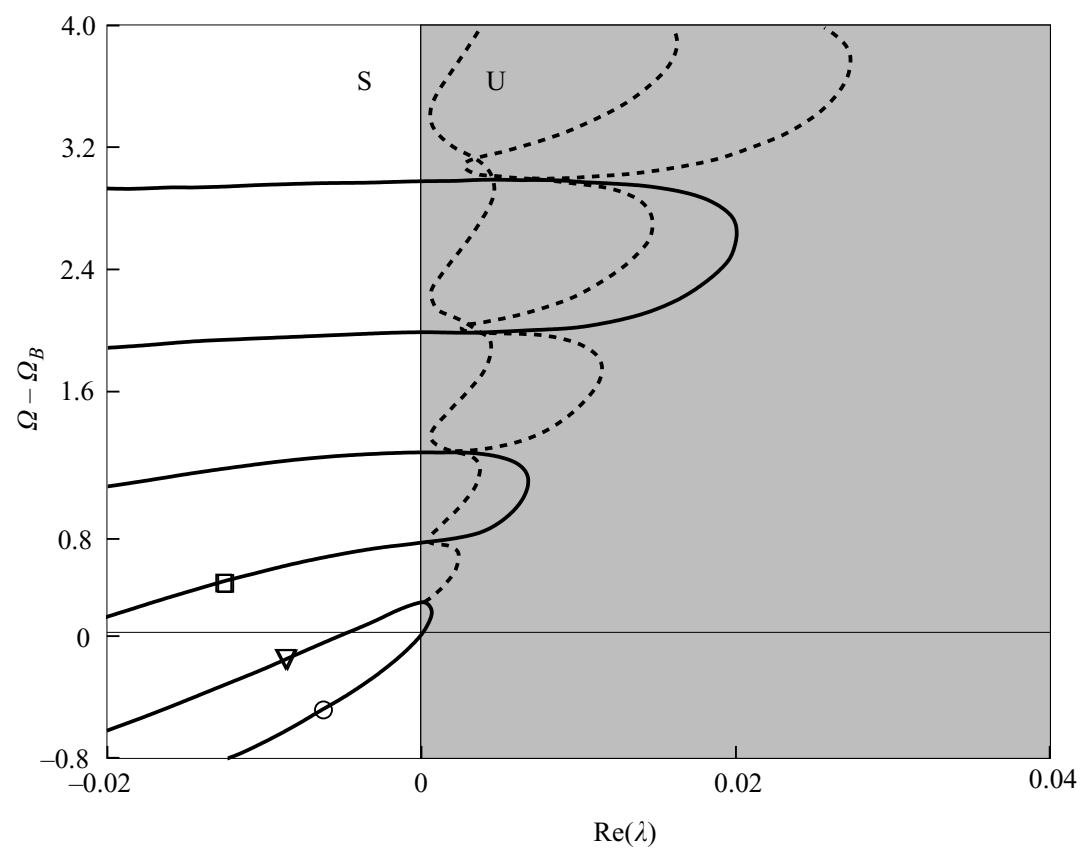

FIGURE 4. Growth rate $\operatorname{Re}(\lambda)$ of most unstable eigenvalues as a function of swirl parameter $\Omega-\Omega_{B}$. Solid lines refer to real eigenvalues and dashed lines to complex-conjugate pairs of eigenvalues. Symbols on curves refer to eigenvalues plotted in figure 3.

which leads to the inhomogeneous system

$$
\left[\begin{array}{cc}
1 & 0 \\
0 & b_{11}^{2}-\frac{\partial^{2}}{\partial z^{2}}
\end{array}\right]\left(\begin{array}{l}
\dot{K} \\
\dot{\psi}
\end{array}\right)=\left[\begin{array}{cc}
-\frac{\partial}{\partial z} & \sqrt{\Omega} \frac{\partial}{\partial z} \\
\sqrt{\Omega} \frac{\partial}{\partial z} & \frac{\partial^{3}}{\partial z^{3}}-b_{11}^{2} \frac{\partial}{\partial z}
\end{array}\right]\left(\begin{array}{c}
K \\
\psi
\end{array}\right)+\left(\begin{array}{c}
-1 \\
0
\end{array}\right) \dot{U}(t),
$$

with homogeneous boundary conditions:

$$
K(0, t)=0, \quad \psi(0, t)=0, \quad \psi_{z z}(0, t)=0, \quad \psi_{z}(L, t)=0 .
$$

The unforced system with non-homogeneous boundary conditions has been transformed into a forced system with homogeneous boundary conditions, a formulation which is particularly suited to the application of control theory. System (4.2)-(4.3) may indeed be cast into the standard form

$$
\mathscr{E} \dot{\boldsymbol{X}}=\mathscr{A} \boldsymbol{X}+\mathscr{B} \boldsymbol{u},
$$

where $\mathscr{E}, \mathscr{A}$ and $\mathscr{B}$ are linear operators, $\boldsymbol{X}=(K, \psi)^{T}$ is the state vector and $\boldsymbol{u}$ is the control vector, which in the presence of a single actuator equals a scalar, $\boldsymbol{u}=\dot{U}$. The field variables $K, \psi$ should not be mistaken for the original full circulation and streamfunction introduced in the Squire-Long equations (2.2-2.4).

Let

$$
\left\langle\boldsymbol{X}_{\alpha}, \boldsymbol{X}_{\beta}\right\rangle=\int_{0}^{L}\left(K_{\alpha}(z) K_{\beta}(z)+\psi_{\alpha}(z) \psi_{\beta}(z)\right) \mathrm{d} z
$$


denote the canonical Cartesian scalar product associated with the $L_{2}$ norm in state space and

$$
\left(\boldsymbol{u}_{\alpha}, \boldsymbol{u}_{\beta}\right)=\boldsymbol{u}_{\alpha}^{*} \boldsymbol{u}_{\beta}
$$

denote the canonical scalar product in control signal space. We also need to introduce the energy norm associated with the expression for the kinetic energy in primitive velocity variables. Integration by parts and use of the boundary conditions (4.3) leads to express the kinetic energy in terms of the so-called 'energetic' scalar product

$$
\left\langle\boldsymbol{X}_{\alpha}, \boldsymbol{X}_{\beta}\right\rangle_{E} \equiv\left\langle\boldsymbol{X}_{\alpha}, \mathscr{E} \boldsymbol{X}_{\beta}\right\rangle,
$$

which only involves the state vector $\boldsymbol{X}=(K, \psi)^{T}$.

Optimal control theory may then be based on the minimization of a cost-functional

$$
\mathscr{J}=\int_{0}^{T}\left(\langle\boldsymbol{X}, \mathscr{E} \boldsymbol{X}\rangle+\ell^{2}(\boldsymbol{u}, \boldsymbol{u})\right) \mathrm{d} t
$$

over the time interval $0 \leqslant t \leqslant T$, where $T$ is the time horizon and the tuning parameter $\ell$ is the so-called control penalty (Zhou, Doyle \& Glover 1995). The cost-functional $\mathscr{J}$ combines the disturbance energy and the cost of the control. If $\ell$ is chosen to be large, the control is 'expensive', if it is small, the control is 'cheap'. In other words, the parameter $\ell$ weighs the control cost with respect to the state energy.

\subsection{Reduced-order system}

Different strategies may be used to minimize $\mathscr{J}$ : one may stay within a continuous formulation and adopt an adjoint formulation (see e.g. Andersson, Berggren \& Henningson 1999; Corbett 2000). As discussed in the introduction, we choose instead to resort to a low-order reduced dynamical system derived from the continuous one by a Galerkin-type expansion. Consider a finite number $N$ of the eigenvectors $\boldsymbol{X}_{i}(z)$ and associated eigenvalues $\lambda_{i}$ determined in $\S 3$. The state $X(z, t)$ is then expanded according to

$$
\boldsymbol{X}(z, t)=\sum_{i=1}^{N} \alpha_{i}(t) \boldsymbol{X}_{i}(z),
$$

where the amplitude functions $\alpha_{i}(t)$ are at this stage unknown and the eigenmodes $\boldsymbol{X}_{i}(z)$ have been normalized so that $\left\langle\boldsymbol{X}_{i}, \boldsymbol{X}_{i}\right\rangle_{E}=1$. Introducing this expression into equation (4.4) leads to

$$
\mathscr{E} \sum_{i=1}^{N} \dot{\alpha}_{i} \boldsymbol{X}_{i}=\mathscr{A} \sum_{i=1}^{N} \alpha_{i} \boldsymbol{X}_{i}+\mathscr{B} \boldsymbol{u} .
$$

The eigenvalues $\lambda_{i}$ satisfy by definition the generalized eigenvalue problem derived from (4.4)

so that (4.10) becomes

$$
\mathscr{A} \boldsymbol{X}_{i}=\mathscr{E} \lambda_{i} \boldsymbol{X}_{i},
$$

$$
\mathscr{E} \sum_{i=1}^{N} \dot{\alpha}_{i} \boldsymbol{X}_{i}=\mathscr{E} \sum_{i=1}^{N} \alpha_{i} \lambda_{i} \boldsymbol{X}_{i}+\mathscr{B} \boldsymbol{u} .
$$

Taking the scalar product of this relation with $\boldsymbol{X}_{j}$ for $j=1 \ldots N$ leads to

$$
\left\langle\boldsymbol{X}_{j}, \mathscr{E} \sum_{i=1}^{N} \dot{\alpha}_{i} \boldsymbol{X}_{i}\right\rangle=\left\langle\boldsymbol{X}_{j}, \mathscr{E} \sum_{i=1}^{N} \alpha_{i} \lambda_{i} \boldsymbol{X}_{i}\right\rangle+\left\langle\boldsymbol{X}_{j}, \mathscr{B}\right\rangle \boldsymbol{u} .
$$




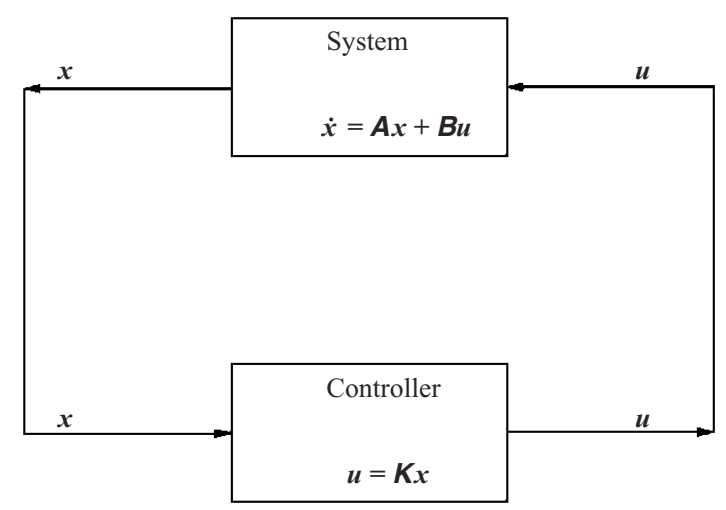

FIGURE 5. Schematic of linear full-information closed-loop control.

Let $\boldsymbol{x}=\left(\alpha_{1}, \ldots, \alpha_{N}\right)^{T}$ denote the reduced-state vector,

$$
\boldsymbol{A}=\left(\begin{array}{ccc}
\lambda_{1} & & 0 \\
& \ddots & \\
0 & & \lambda_{N}
\end{array}\right)
$$

denote the diagonal matrix of eigenvalues and $\boldsymbol{M}$ denote the square 'matrix of angles', with elements $M_{i j}=\left\langle\boldsymbol{X}_{j}, \mathscr{E} \boldsymbol{X}_{i}\right\rangle$. The projected system (4.13) may then be written in the form

$$
\boldsymbol{M} \dot{\boldsymbol{x}}=\boldsymbol{M A x}+\left\langle\boldsymbol{X}_{j}, \mathscr{B}\right\rangle \boldsymbol{u} .
$$

Multiplying both sides by $\boldsymbol{M}^{-1}$ finally leads to the compact notation

$$
\dot{\boldsymbol{x}}=\boldsymbol{A x}+\boldsymbol{B u},
$$

where the 'receptivity' matrix $\boldsymbol{B}$ of dimension $N \times 1$ is by definition $\boldsymbol{B}=\boldsymbol{M}^{-1}\left\langle\boldsymbol{X}_{j}, \mathscr{B}\right\rangle$. The reduced discrete dynamical system (4.15) is cast in a form that is directly amenable to classical optimal control theory as outlined in the next section.

\subsection{Application of optimal control theory}

Figure 5 summarizes the concept of linear closed-loop control applied to the discrete system (4.15). The control amplitude $\boldsymbol{u}(t)$ is sought as a linear function of the state vector $\boldsymbol{x}$ according to

$$
\boldsymbol{u}=\boldsymbol{K} \boldsymbol{x}
$$

where $\boldsymbol{K}(t)$ is an unknown gain matrix. Optimal control theory (also called LQR or $\mathscr{H}_{2}$ control) provides a way to select the 'best' gain matrix $\boldsymbol{K}$ in order to minimize the discrete counterpart of the cost-functional (4.8) at time horizon $T$. When expressed in the discrete state space $\boldsymbol{x}$, the cost function becomes

$$
J=\int_{0}^{T}\left(\boldsymbol{x}^{*} \boldsymbol{M} \boldsymbol{x}+\ell^{2} \boldsymbol{u}^{*} \boldsymbol{u}\right) \mathrm{d} t
$$

where ${ }^{*}$ denotes the transconjugate and $\boldsymbol{x}^{*} \boldsymbol{M} \boldsymbol{x}$ is the discretized energy norm of $\boldsymbol{x}$ in the sense of (4.7). When in addition the time horizon $T$ is set to infinity, standard optimal control theory, as outlined in Zhou et al. (1995), yields the following two key results:

the gain matrix $\boldsymbol{K}$ becomes independent of time; 

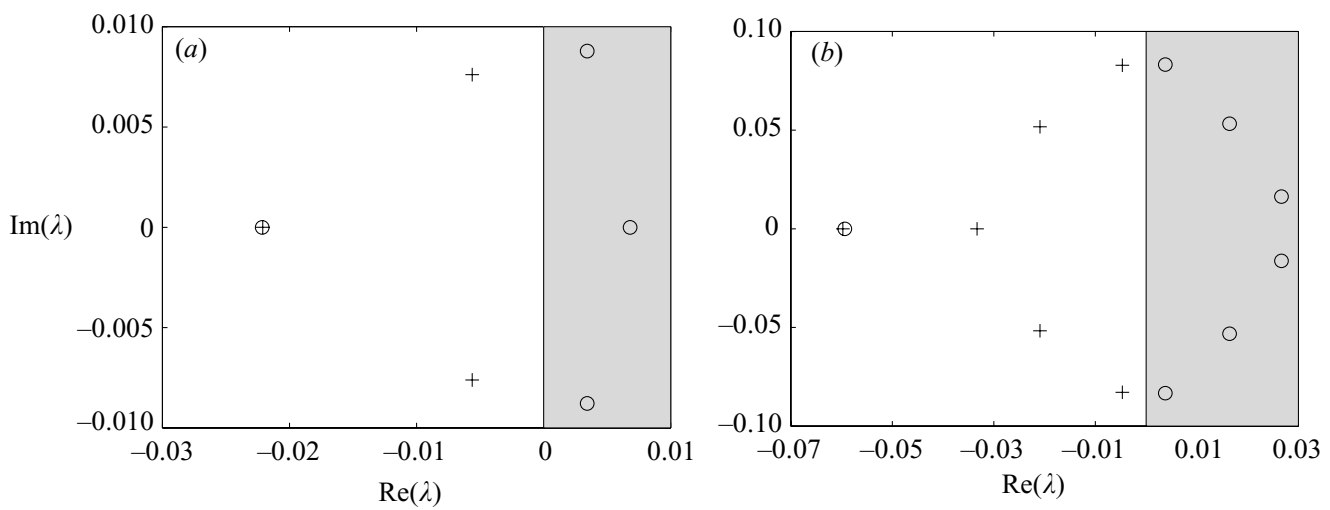

FiguRE 6. Effect of optimal closed-loop control on the most unstable eigenvalues of the reduced-order system; O, eigenvalues without control; + , eigenvalues with control. (a) $\Omega=$ $\Omega_{B}+1 ;(b) \Omega=\Omega_{B}+4$; in both cases $\ell=1$.

the solution to the optimization problem is found by solving the Riccati equation for $Y$

$$
\boldsymbol{A}^{*} \boldsymbol{Y}+\boldsymbol{Y} \boldsymbol{A}-\boldsymbol{Y} \boldsymbol{B} \boldsymbol{B}^{*} \boldsymbol{Y} / \ell^{2}+\boldsymbol{M}=0,
$$

and then selecting the gain matrix to be

$$
\boldsymbol{K}=-\boldsymbol{B}^{*} \boldsymbol{Y} / \ell^{2} .
$$

The Riccati equation (4.18) is solved by using an eigenvalue decomposition (Laub 1991) and it is implemented in matlab.

The results of the low-order optimal control scheme in the subspace spanned by the eigenmodes $\boldsymbol{X}_{i}(z), i=1 \ldots N$ are displayed in figure 6 . The eigenvalues of the uncontrolled system (4.15) (with $\boldsymbol{u}=0$ ) are nothing more than the eigenvalues of the matrix $\boldsymbol{A}$. The eigenvalues of the controlled system (4.15) (with $\boldsymbol{u}=\boldsymbol{K} \boldsymbol{x}$ ) are those of the matrix $\boldsymbol{A}+\boldsymbol{B} \boldsymbol{K}$. These two sets are compared in figure 6 for the two swirl parameter settings $\Omega=\Omega_{B}+1$ and $\Omega=\Omega_{B}+4$, when the truncation order is $N=12$ and the control penalty is $\ell=1$. In the case of figure $6(a)\left(\Omega=\Omega_{B}+1\right)$, the three unstable modes are effectively shifted to the stable left-hand-side plane. Similarly in figure $6(b)\left(\Omega=\Omega_{B}+4\right)$, the six unstable modes are quenched. It is important to note that the selected actuator remains effective at $\Omega=\Omega_{B}+15$, i.e. $100 \%$ above threshold, at which stage all the $N=12$ modes are unstable.

\section{Application of the reduced-order optimal control scheme to the full linear instability equations}

In order to verify the efficiency of the low-order control loop designed in the preceding section, the evolution equations (4.2)-(4.3) are solved numerically. The continuous state vector $\boldsymbol{X}(z, t)=(K(z, t), \psi(z, t))^{T}$ is replaced by its discrete analogue, a columnar vector

$$
x(t)=\left(K\left(z_{1}, t\right), \ldots, K\left(z_{q}, t\right), \psi\left(z_{1}, t\right), \ldots, \psi\left(z_{q}, t\right)\right)^{T}
$$

made up of the values taken by $K(z, t)$ and $\psi(z, t)$ at the $q$ collocation points $z_{1}, \ldots, z_{q}$. A standard Chebyshev-collocation technique described, for instance, in Schmid \& Henningson (2001), is implemented with the help of matlab. The collocation points $z_{1}, \ldots, z_{q}$ on the interval $0 \leqslant z \leqslant L$ are mapped onto the Gauss-Lobatto 
collocation points $z_{j}^{\prime}=\cos (\pi j / q)$ on the interval $-1 \leqslant z^{\prime} \leqslant 1$. The differential operators are thereby converted into their matrix counterparts and the boundary conditions at $z=0$ and $z=L$ are handled in the same fashion as in Schmid \& Henningson (2001). This procedure leads to the discrete dynamical system

$$
E \dot{X}=A X+B \dot{U},
$$

which is a discrete representation of the continuous system (4.4). In practice, it is sufficient to choose $q=60$ Chebyshev collocation points in order to recover the generalized eigenvalues directly computed in $\S 3$ from the roots of $D(\lambda)=0$. As illustrated in figure 3 , the five leading eigenvalues independently calculated via these two procedures are found to be identical. One should emphasize that searching for the eigenvalues associated to (5.1) also yields spurious unstable eigenvalues which can easily be recognized since they do not converge to any definite value as the truncation parameter $q$ is increased. In order to filter them out, an Euler backward implicit time scheme with $\Delta t=0.2$ is implemented to track the temporal evolution of the dynamical system (5.1). Initial conditions are taken to be

$$
K(z, 0)=\sin \left(\frac{\pi z}{2 L}\right), \quad \psi(z, 0)=\sin \left(\frac{\pi z}{2 L}\right) .
$$

From now on, we do not distinguish between the continuous system (4.4) and its discrete counterpart (5.1). For our purposes, these two systems are deemed to be equivalent and we present the formulation only in the context of the continuous system (4.4). It is understood that the calculations are carried out on the discrete representation.

In the previous section, an optimal gain matrix $\boldsymbol{K}$ was determined in order to control the time evolution of the reduced-state vector $\boldsymbol{x}=\left(\alpha_{1}, \alpha_{2}, \ldots \alpha_{N}\right)^{T}$. The objective is now to exploit this result to control the full linear system (4.4). The missing link is the connection between the full-state vector $\boldsymbol{X}(z, t)$ and the reduced-state vector $\boldsymbol{x}(t)$. In other words, we seek the projection operator $\Pi$ which projects $X$ onto the subspace spanned by the $N$ eigenmodes $\left\{\boldsymbol{X}_{i}(z)\right\}$ of $\S 4$, via the relation

$$
\boldsymbol{x}(t)=\Pi \boldsymbol{X}(z, t) .
$$

Note that the projector $\Pi$ is not unique: any scalar product in the full-state space leads to an orthogonal projection of $\boldsymbol{X}$ onto this subspace. In the present analysis, we choose the scalar product \langle\rangle defined in relation (4.5) and the state vector $\boldsymbol{X}$ is decomposed into

$$
\boldsymbol{X}=\sum_{i=1}^{N} \alpha_{i} \boldsymbol{X}_{i}+R,
$$

where the remainder $R$ is such that $\left\langle\boldsymbol{X}_{i}, R\right\rangle=0$, for all $i$. Taking the Cartesian scalar product with the $\boldsymbol{X}_{j}$ yields

$$
\left\langle\boldsymbol{X}_{j}, \boldsymbol{X}\right\rangle=\sum_{i=1}^{N} \alpha_{i}\left\langle\boldsymbol{X}_{j}, \boldsymbol{X}_{i}\right\rangle .
$$

Let $\mathscr{P}_{2}$ be the $N$-component operator such that $\mathscr{P}_{2} \boldsymbol{X} \equiv\left\langle\boldsymbol{X}_{j}, \boldsymbol{X}\right\rangle$ and $\mathbf{M}_{2}$ the $N \times N$ matrix of angles $\left\langle\boldsymbol{X}_{j}, \boldsymbol{X}_{i}\right\rangle$. Expression (5.5) then becomes

$$
\mathscr{P}_{2} \boldsymbol{X}=\mathbf{M}_{2} \boldsymbol{x},
$$




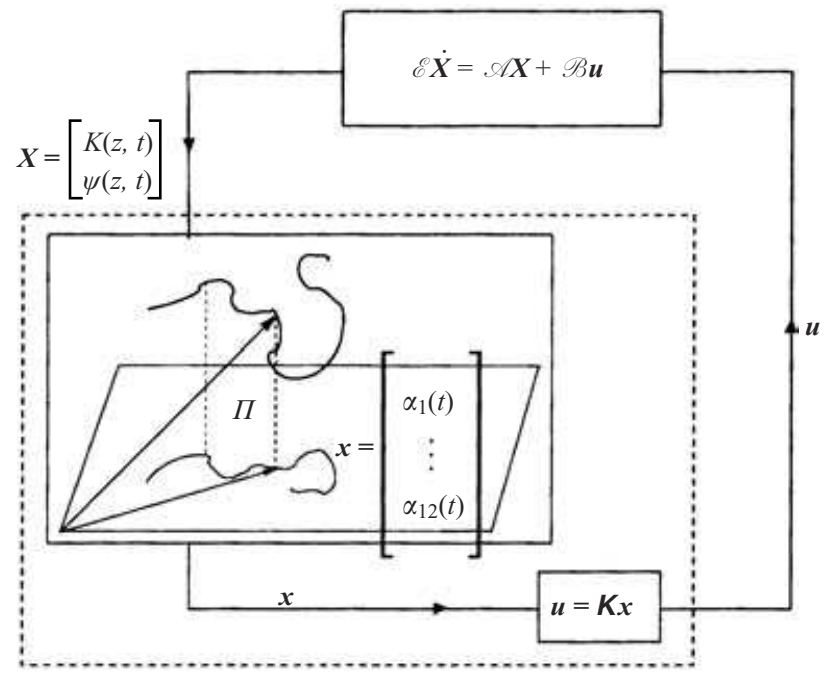

FiguRE 7. Full-state control scheme: the full-state vector $\boldsymbol{X}$ emerging from the plant is projected on the subspace spanned by the first $N$ linear eigenmodes by means of the projection operator $\Pi$, to yield the reduced-state vector $\boldsymbol{x}$. The control given by $\boldsymbol{u}=\boldsymbol{K} \boldsymbol{x}$, where $\boldsymbol{K}$ is the reduced-order optimal gain matrix, is then fed back into the full plant.

whereby the projector $\Pi$ is finally given by

$$
\Pi=\boldsymbol{M}_{2}^{-1} \mathscr{P}_{2} .
$$

Note that the matrix $\boldsymbol{M}_{2}$ can be calculated once and for all, whereas $\mathscr{P}_{2}$ and therefore $\Pi$ are formally continuous operators acting on the full-state vector $X(z, t)$. In the discrete representation outlined at the beginning of this section, $\mathscr{P}_{2}$ and $\Pi$ become $N \times 2 q$ matrices.

The control scheme pertaining to the full state system is sketched in figure 7. Given the plant $\mathscr{E} \dot{\boldsymbol{X}}=\mathscr{A} \boldsymbol{X}+\mathscr{B} \boldsymbol{u}$, the full state $\boldsymbol{X}$ is projected as $\boldsymbol{x}$ onto the reduced subspace, via the projector $\Pi$. From the reduced state $\boldsymbol{x}$, one determines the optimal control $\boldsymbol{u}=\boldsymbol{K} \boldsymbol{x}$, with $\boldsymbol{K}$ given in $\S 4$, which is fed back into the plant.

The results of the procedure are displayed in figure 8 for the swirl parameter setting $\Omega=\Omega_{B}+1$, with $N=12$ and $\ell=1$. Recall that in such a case (see figure $6 a$ ), there are three unstable eigenvalues. In the uncontrolled situation $(\boldsymbol{u}=0)$, the full-state kinetic energy (solid line)

$$
E(t)=\langle\boldsymbol{X}, \mathscr{E} \boldsymbol{X}\rangle \equiv\langle\boldsymbol{X}, \boldsymbol{X}\rangle_{E}
$$

increases exponentially with twice the slope of the real part of the most unstable eigenvalue $\lambda_{1}$. Upon application of the control, the full-state energy (dashed line) is driven to zero exponentially fast. The control energy (dotted line) $(\boldsymbol{u}, \boldsymbol{u})$ remains bounded, and even decays exponentially with time. These computations constitute the main result of the present study: the effectiveness of a reduced-order control scheme has been demonstrated on the full linear system describing the evolution of unstable modes on columnar vortices.

A less favourable case is illustrated in figure 9 at the higher level of supercriticality $\Omega=\Omega_{B}+4$, with the same settings for $N$ and $\ell$. There are now six unstable eigenvalues 


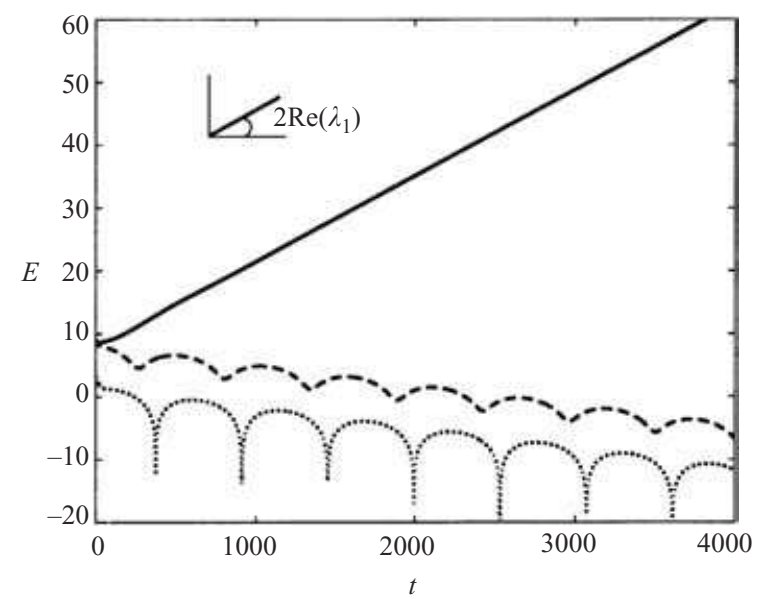

Figure 8. Perturbation energy $E$ as a function of time $t$ on a semi-log plot for $\Omega=\Omega_{B}+1$, $N=12, \ell=1$. Solid line: no control, dashed line: with control; dotted line: control energy.

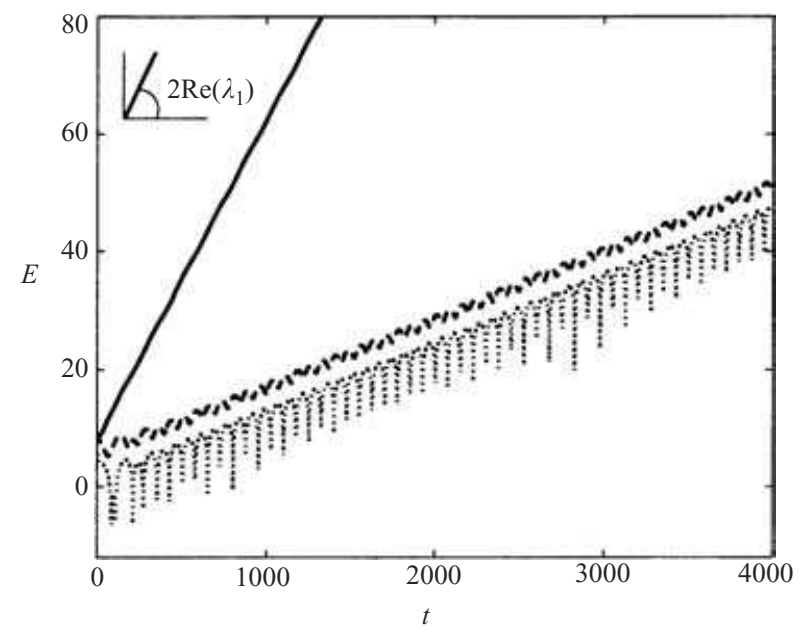

Figure 9. Perturbation energy $E$ as a function of time $t$ on a semi-log plot for $\Omega=\Omega_{B}+4$, $N=12, \ell=1$. Solid line: no control, dashed line: with control; dotted line: control energy.

(figure $6 b$ ). The perturbation kinetic energy $E(t)$ with control is seen to grow exponentially, albeit at a slower growth rate than in the uncontrolled system. In contrast with the previous situation, the control energy keeps growing exponentially: the reduced-order control $\boldsymbol{u}$ is unable to quench the instability. The effective controllability limit has been determined, by trial and error, to be $\Omega_{c}=\Omega_{B}+1.9$, i.e. $13 \%$ above the critical swirl $\Omega_{1}$. At this stage, there are four unstable modes.

\section{Parametric study and robustness issues}

\subsection{Effect of the control penalty $\ell$}

The control penalty $\ell$ is the weighting factor of the control cost appearing in the functional $\mathscr{J}$ defined in (4.8). It determines if the control is expensive (large $\ell$ ) or cheap (small $\ell$ ). In the reduced space, where optimal control theory is applied, the 

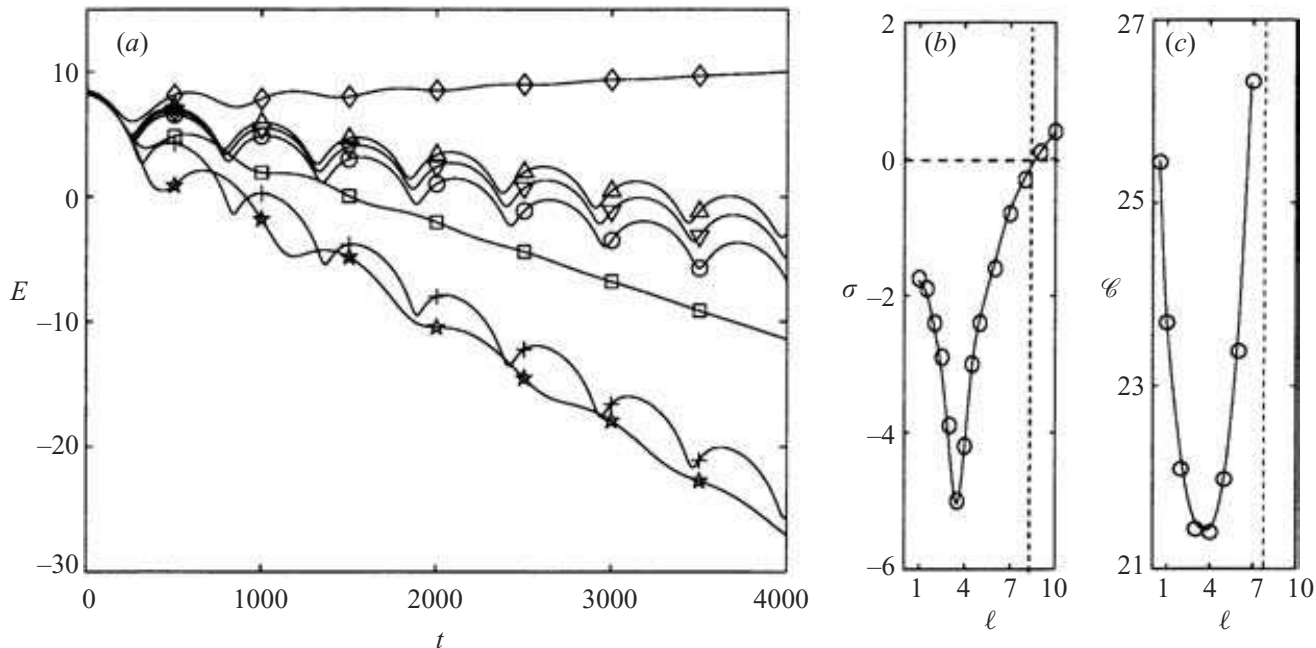

FiguRE 10. (a) Temporal evolution of the controlled-state energy $E$ on a semi-log plot for $\Omega=\Omega_{B}+1$ and different values of the control penalty $\ell: \ell=10(\diamond) ; \ell=5(\square) ; \ell=4(\star) ; \ell=3(+)$; $\ell=1(\circ) ; \ell=0.5(\nabla) ; \ell=0.1(\triangle)$. (b) Asymptotic growth rate $\sigma$ of the controlled-state energy versus control penalty $\ell$. (c) Control cost $\mathscr{C}$ at time horizon $T=4000$ as a function of $\ell$.

control stabilizes the system whatever value of $\ell$ is chosen, as predicted by optimal control theory. It remains to determine the influence of the $\ell$ parameter on the loworder control of the full system. Will the projection method work for any value of $\ell$ ? Will a small value of $\ell$ stabilize the full state more effectively than a larger one? Some clues may be found in figure 10(a), which depicts the temporal evolution of the controlled-state energy on a semi-log plot for different values of $\ell$ at the same swirl $\Omega=\Omega_{B}+1$.

The resulting asymptotic growth rates of the state energy are displayed in figure $10(b)$, as a function of the cost penalty $\ell$. For this particular value of $\Omega$, the feedback control is seen to lose its effectiveness as soon as $\ell \geqslant 8$. In other words, the reduced-order control has to be cheap enough and therefore potentially large enough in order to work well in the full-state space. Figure $10(b)$ indicates that the most efficient control is not necessarily the cheapest; there is an optimal value $\ell \sim 4$ maximizing the decay rate of the state energy. The cost of the control $\mathscr{C}=\int_{0}^{\infty} \boldsymbol{u}^{*} \boldsymbol{u} \mathrm{d} t$ is represented as a function of $\ell$ in figure $10(c)$. In practice the time integration is stopped at $T=4000$, a value which is large enough so that the time-integral defining $\mathscr{C}$ has reached an asymptotic plateau for all $\ell$-values of interest. The most efficient stabilization (at $\ell=4$ ) is seen to be obtained for the minimum cost. This parametric study demonstrates that the most efficient control should be designed with $\ell=4$, at least at this swirl setting.

\subsection{Effect of the reduction order $N$}

According to the previous section, there are four unstable eigenvalues at the effective controllability limit $\Omega_{c}$. This observation suggests that it may be possible to decrease the reduced-space dimension $N$ to the four most unstable modes, without compromising controllability. The effect of $N$ on the temporal evolution of the controlled-state energy is displayed in figure 11, at the swirl parameter $\Omega=\Omega_{B}+1.7$, in which case four modes are unstable. Surprisingly, $N=4$ is nearly as effective as $N=12$ in stabilizing the system. However as $N$ is decreased to 3 , the flow cannot be 


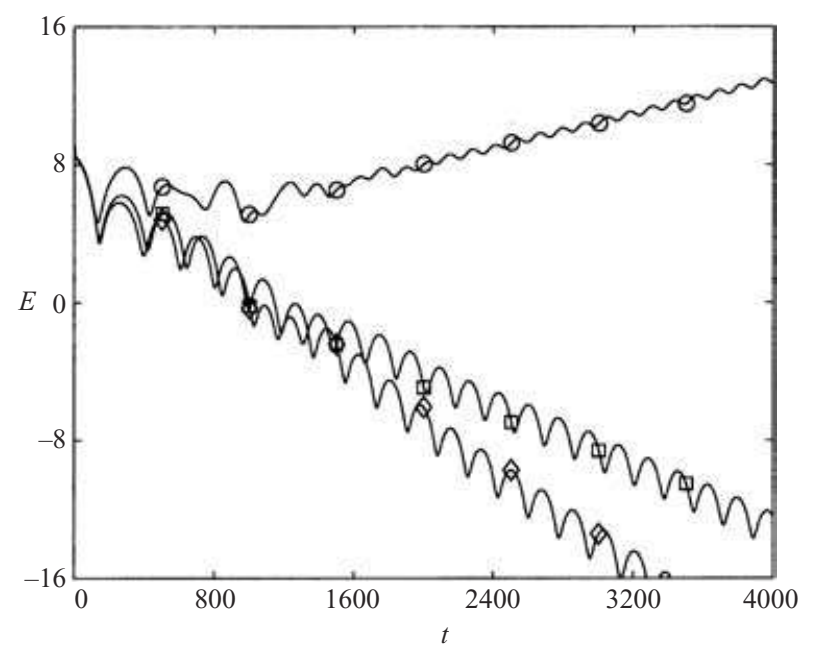

FIGURE 11. Effect of the reduced system order $N$. Perturbation energy $E$ as a function of time $t$ on a semi-log plot for $\Omega=\Omega_{B}+1.7, \ell=5$ and different values of $N: N=12(\diamond), N=4(\square)$ and $N=3(\circ)$.

controlled: in this instance, the design of the control scheme has failed to account for one of the four unstable modes!

\subsection{Robustness considerations}

The robustness of the control scheme can be heuristically tested by adding a random noise at each time step with the same mean energy as the initial condition (5.2). The noise is selected to have a standard deviation of $36 \%$ of its mean energy. As seen in figure 12 for $\Omega=\Omega_{B}+1$, the control remains effective since the controlled-state energy remains bounded. Note, however, that it is no longer driven to zero, as in figure 8 in the absence of noise. The controlled-state energy is seen to oscillate around a mean value of about 3600 times the noise energy with a standard deviation of $105 \%$. Although the controlled system does not diverge, it amplifies external noise both in the mean response and in the intensity of the oscillations about the mean.

Robustness with respect to uncertainties in control-parameter settings, as defined in Or, Cortelezzi \& Speyer (2001) may also be tested. First a control is designed for a nominal parameter value, say $\Omega_{p}$, and the effective control range is then defined as the interval in $\Omega$ where the control designed for $\Omega_{p}$ still stabilizes the flow at $\Omega$. Results are displayed in figure 13. The control range, represented by a vertical segment, is plotted as a function of the nominal parameter value $\Omega_{p}-\Omega_{B}$. The two vertical solid lines correspond, respectively, to the instability onset at $\Omega-\Omega_{B}=\pi^{2} / 4 L^{2} \sim 0.02$ to the left and to the effective controllability limit of $13 \%$ to the right. The control range is seen to present strong non-uniformities but there are only very few nominal swirls $\Omega_{p}$ where it shrinks down to a single point. This graph may also be interpreted in another way: for a given operational swirl $\Omega-\Omega_{B}$ along the vertical axis, the shaded area selects a horizontal segment characterizing the swirl measurement error which can be tolerated without compromising control effectiveness. The above considerations indicate that the control generally remains effective even if the swirl setting is offdesign. 


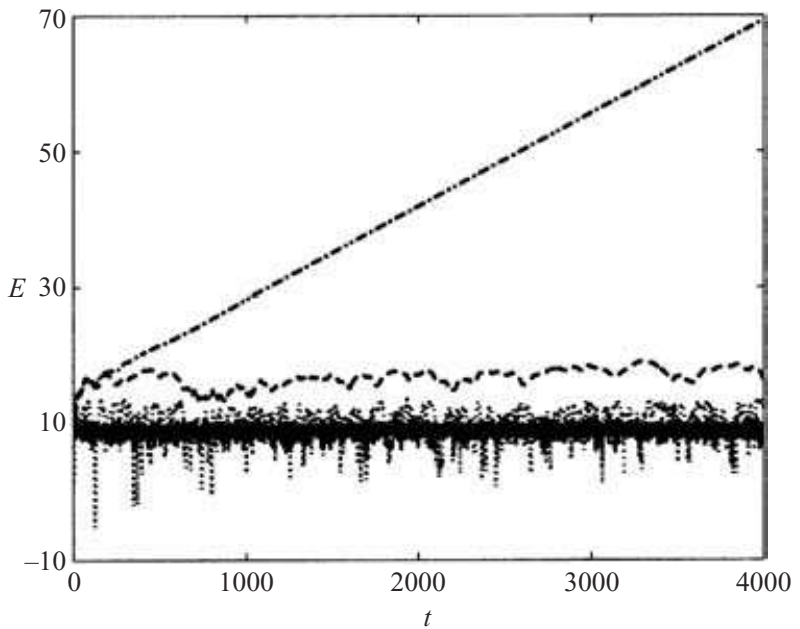

FIGURE 12. Robustness of control scheme with respect to noise: perturbation energy $E$ as a function of time $t$ on a semi-log plot for $\Omega=\Omega_{B}+1, N=12$ and $\ell=1$. Random noise of the same energy as the initial condition is added at each time step, with a standard deviation of $36 \%$ and spatially distributed over the first $N$ eigenmodes. Solid line: noise energy; dotted-dashed line: no control; dashed line: with control; dotted line: control energy.

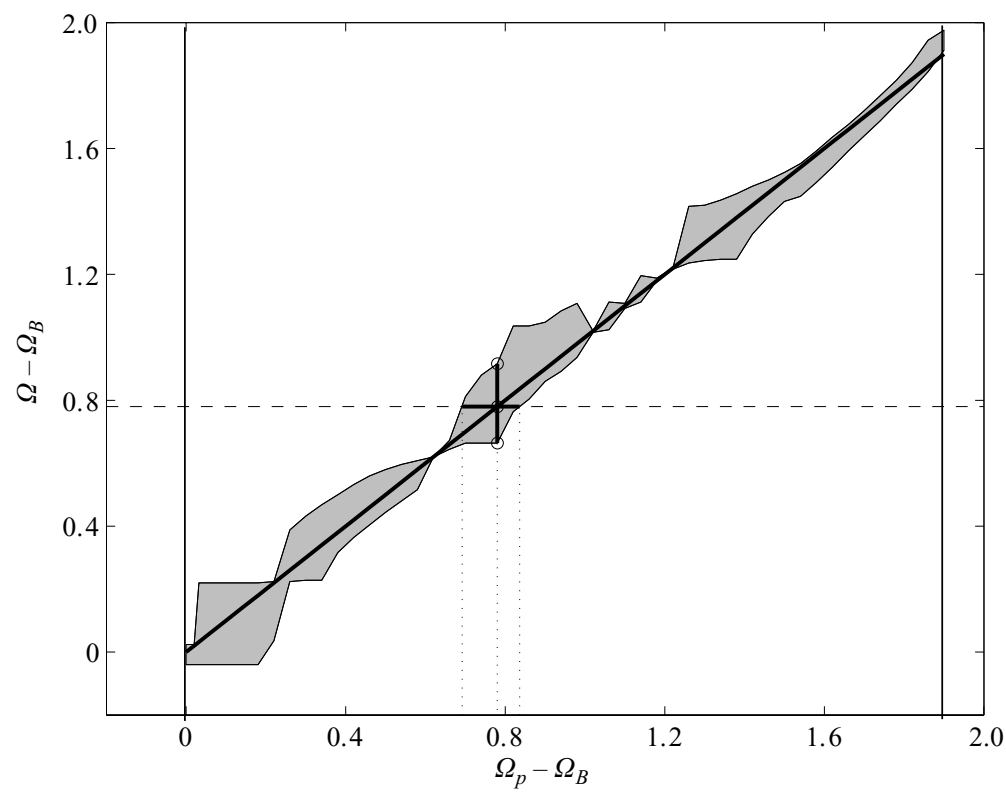

FIGURE 13. Robustness of control scheme with respect to uncertainties in parameter setting $\Omega$. Effective control range, defined in terms of the $\Omega-\Omega_{B}$ interval as a function of nominal design swirl number $\Omega_{p}-\Omega_{B}$ for $N=12, \ell=1$.

\subsection{Driving the 'true' control to zero}

It should be emphasized that throughout the analysis of the previous sections, the control variable $\boldsymbol{u}=\dot{U}$ has been defined as the time derivative of the 'true' control signal $U(t)$. Furthermore, the control $\boldsymbol{u}$ was designed to minimize the functional $\mathscr{J}$ 


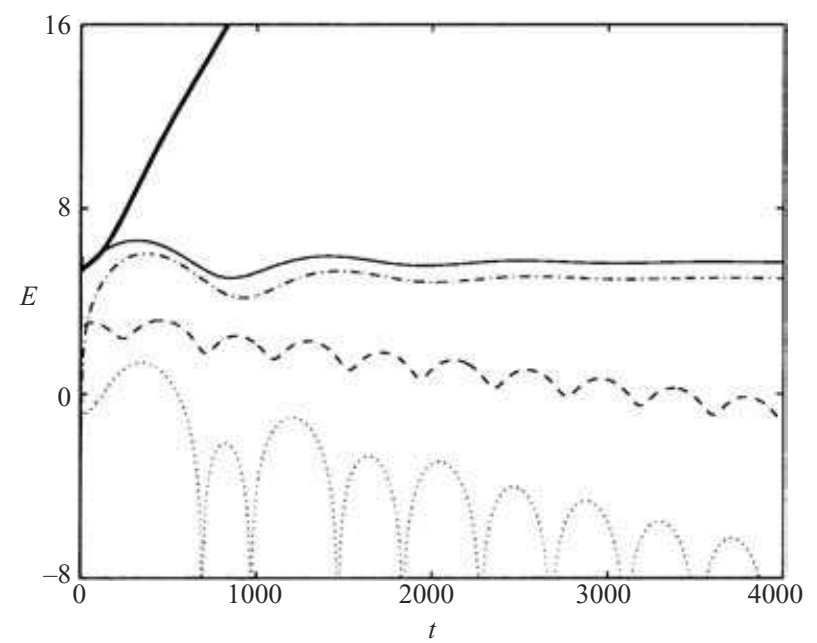

FIGURE 14. Evolution of 'true' energies $E^{1}=\left\langle\boldsymbol{X}^{1}, \mathscr{E} \boldsymbol{X}^{1}\right\rangle$ and $\|U\|^{2}$ pertaining to original variables $K^{1}(z, t), \psi^{1}(z, t)$ and $U(t)$ introduced in (4.1) on a semi-log plot as a function of time for $\Omega=\Omega_{B}+1, N=12$ and $\ell=1$. Thick solid line: $E^{1}(t)$ without control; thin solid line: $E^{1}(t)$ with control and without augmentation; dashed-dotted line: $\|U\|^{2}(t)$ without augmentation; dashed line: $E^{1}(t)$ with control and with augmentation $\left(r=10^{3}\right)$; dotted line: $\|U\|^{2}(t)$ with augmentation.

defined in (4.8) as the time-integral of the sum of the state energy $E(t)=\langle\boldsymbol{X}, \mathscr{E} \boldsymbol{X}\rangle$ and the control energy $(\boldsymbol{u}, \boldsymbol{u})$. This procedure demonstrated that both quantities decrease exponentially to zero with time when control is applied. It remains to establish that the 'true' energy $E^{1}(t)=\left\langle\boldsymbol{X}^{1}, \mathscr{E} \boldsymbol{X}^{1}\right\rangle$, where $X^{1}(z, t)=\left(K^{1}(z, t), \psi^{1}(z, t)\right)^{T}$ denotes the vector before the change of variables (4.1), and the 'true' control energy $\|U\|^{2}$ also decay to zero with time. Both these 'true' measures are represented in figure 14 , in the case where the initial value of $U(t)$ has been set to $U(0)=0$. It is noteworthy that $E^{1}(t)$ and $\left\|U^{2}\right\|(t)$ do not decay to zero: rather, they tend to constant values for large time. One might argue that the initial value $U(0)$ may be chosen $a$ posteriori in such a way that $E^{1}(t)$ and $\left\|U^{2}\right\|(t)$ go to zero. We look instead for an a priori method based on the augmentation of the state space, as suggested in the fluid mechanical context by Joshi et al. (1997) and Högberg et al. (2003). Accordingly, let the full-state system (4.4) be replaced by the augmented state-space form

$$
\left[\begin{array}{cc}
\mathscr{E} & 0 \\
0 & 1
\end{array}\right]\left(\begin{array}{c}
\dot{\boldsymbol{X}} \\
\dot{U}
\end{array}\right)=\left[\begin{array}{cc}
\mathscr{A} & 0 \\
0 & 0
\end{array}\right]\left(\begin{array}{l}
\boldsymbol{X} \\
U
\end{array}\right)+\left(\begin{array}{c}
\mathscr{B} \\
1
\end{array}\right) \boldsymbol{u},
$$

the low-order system (4.15) be replaced by

$$
\left[\begin{array}{cc}
\boldsymbol{I} & 0 \\
0 & 1
\end{array}\right]\left(\begin{array}{c}
\dot{\boldsymbol{x}} \\
\dot{U}
\end{array}\right)=\left[\begin{array}{cc}
\boldsymbol{A} & 0 \\
0 & 0
\end{array}\right]\left(\begin{array}{l}
\boldsymbol{x} \\
U
\end{array}\right)+\left(\begin{array}{l}
\boldsymbol{B} \\
1
\end{array}\right) \boldsymbol{u},
$$

and the cost functional (4.8) by

$$
\mathscr{J}=\int_{0}^{T}\left(\boldsymbol{x}^{*} \boldsymbol{M} \boldsymbol{x}+\ell^{2} \boldsymbol{u}^{*} \boldsymbol{u}+\left(1+r^{2}\right)\|U\|^{2}\right) \mathrm{d} t .
$$

The addition of the last term in the integrand of (6.3) ensures that $U(t)$ also is minimized. As advocated by Högberg \& Henningson (2002), a second tuning 
parameter $r$ has been introduced in order to stabilize the zero eigenvalue in the augmented matrix (6.2). We consequently apply the same methodology consisting of a control design on the reduced-order system (6.2) followed by a projection procedure defined in (5.3)-(5.7) and adapted to the augmented state vector $(\boldsymbol{X}, U)^{T}$. Typical results of the augmentation procedure with $\ell=1$ and $r=10^{3}$ are illustrated in figure 14. The 'true' state energy is seen to decrease to zero exponentially, in contrast with its non-augmented counterpart. The same holds for the 'true' control energy.

\section{Actuators and sensors}

\subsection{Choice of actuators}

As stated in $\S 3$, the Wang \& Rusak instability is driven by the inlet and outlet conditions, which are the only energy providers in the flow. This is the primary motivation to place the actuator at the pipe inlet or outlet. In the last three sections, we have designed a full-state information control loop with the actuator placed at the inlet, the instantaneous inlet circulation perturbation being $K^{(1)}(0, t) \equiv U(t)$. Let us now examine the performance of an actuator located at the outlet and controlling the outlet radial velocity through $\psi_{z}^{(1)}(L, t) \equiv U(t)$. In order to cast the forced linear system in a suitable form for the application of optimal control theory, the variables are changed according to

$$
\left(\begin{array}{l}
K^{(1)} \\
\psi^{(1)}
\end{array}\right)=\overbrace{\left(\begin{array}{c}
K \\
\psi
\end{array}\right)}^{X}+\overbrace{\left(\begin{array}{c}
\sqrt{\Omega_{B}} g(z) \\
g(z)
\end{array}\right)}^{G} U(t)
$$

where $g(z)$ is assumed to satisfy

$$
g_{z z z}-\sqrt{\Omega-\Omega_{B}} g_{z}=0,
$$

with boundary conditions

$$
g(0)=0, \quad g_{z z}(0)=0, \quad g_{z}(L)=0 .
$$

The function $g(z)$ is given by

$$
g(z)=a \cos \left(\sqrt{\Omega-\Omega_{B}} z\right)+b \sin \left(\sqrt{\Omega-\Omega_{B}} z\right)+c,
$$

with $a, b$ and $c$ solutions of the linear system

$$
\left(\begin{array}{l}
a \\
b \\
c
\end{array}\right)=\left(\begin{array}{ccc}
1 & 0 & 1 \\
\Omega-\Omega_{B} & 0 & 0 \\
-\sqrt{\Omega-\Omega_{B}} \sin \left(\sqrt{\Omega-\Omega_{B}} L\right) & \sqrt{\Omega-\Omega_{B}} \cos \left(\sqrt{\Omega-\Omega_{B}} L\right) & 0
\end{array}\right)^{-1}\left(\begin{array}{l}
0 \\
0 \\
1
\end{array}\right) .
$$

Upon making this change of variables, the system finally becomes

$$
\mathscr{E} \dot{\boldsymbol{X}}=\mathscr{A} \boldsymbol{X}+\mathscr{B} \boldsymbol{u}
$$

with $\boldsymbol{u}=\dot{U}$ and where the 'receptivity' matrix $\mathscr{B}$ equals

$$
\mathscr{B}=\left(\begin{array}{c}
-\sqrt{\Omega} g(z) \\
-\left(g_{z z}(z)-\Omega_{B} g(z)\right)
\end{array}\right) .
$$

The same strategy as in $\S \S 4$ and 5 can then be followed step-by-step. The effective controllability limit is determined to be $28 \%$ above $\Omega_{B}$, thereby showing that a 


\section{Actuator}

$$
\begin{aligned}
& K(0)=U(t) \\
& \psi(0)=U(t) \\
& \psi_{z z}(0)=U(t) \\
& \psi_{z}(L)=U(t) \\
& {\left[K(0), \psi_{z}(L)\right]=\left[U_{1}(t), U_{2}(t)\right]}
\end{aligned}
$$

Controllability limit (\%)

13

10

6

28

28

TABLE 1. Effective controllability limit for different actuators expressed as a percentage above $\Omega_{B}$.

controller located at the outlet can be much more effective than at the inlet. Similar changes of variables can be derived leading to expressions analogous to (7.6) for an actuator controlling one of the two remaining boundary conditions in (3.3). The results are collected in table 1: the most efficient control is obtained by acting on the radial velocity at the downstream end of the pipe.

A double actuator can also be easily implemented by considering a control amplitude with two degrees of freedom as well as a double column control matrix $\mathscr{B}$; our computations however have shown that the implementation of a double actuator does not further increase the controllability limit.

\subsection{Sensors without estimation}

In the formulation presented in $\S 5$, the reduced-order state $\boldsymbol{x}$ has been obtained by projecting the full state vector $\boldsymbol{X}$. However, in certain cases, it may happen that only partial knowledge of the system is available and, in such situations, the control effectiveness must be ascertained. This is, in particular, the case in physically relevant situations where sensors have to be used to measure the internal state. The general methodology then consists in deriving a Kalman filter to estimate the internal state from the measurements. In the present situation, we can take advantage of the reduced-order control strategy. Let $\boldsymbol{Y}=\mathscr{C} \boldsymbol{X}$ be the partial-state vector available to the designer, where $\mathscr{C}$ is a linear operator. Since we are only interested in retrieving the reduced-order state vector $\boldsymbol{x}$ in order to determine the control $\boldsymbol{u}=\boldsymbol{K} \boldsymbol{x}$, it seems reasonable that partial information may be sufficient to recover $\boldsymbol{x}$, as long as the partial state dimension exceeds the reduced-order $N$ ! To test this conjecture, we set $N=4$ and assume that the component $\psi(z, t)$ of the state $X(z, t)$ is available only at four equally spaced points

$$
\boldsymbol{Y}=(\psi(L / 5, t), \psi(2 L / 5, t), \psi(3 L / 5, t), \psi(4 L / 5, t))^{T} .
$$

Physically, this corresponds to the measurement of the axial velocity perturbation at four axial locations.

As in $\S 5$, the missing link is the projector $\tilde{\Pi}$ which projects the partial-state vector $\boldsymbol{Y}(t)$ onto the reduced subspace spanned by the $N$ eigenmodes $\boldsymbol{X}_{i}(z)$ of $\S 3$ via the relation

$$
\boldsymbol{x}(t)=\tilde{\Pi} \boldsymbol{Y}(t)
$$

The procedure is the same as for the determination of $\Pi$ in $\S 5$. By analogy with the scalar product defined in (4.5), let us first introduce the Cartesian scalar product on the partial state $Y$ according to

$$
\left[\boldsymbol{Y}_{\alpha}, \boldsymbol{Y}_{\beta}\right]=\left\langle\boldsymbol{X}_{\alpha}, \mathscr{C}^{t} \mathscr{C} \boldsymbol{X}_{\beta}\right\rangle
$$




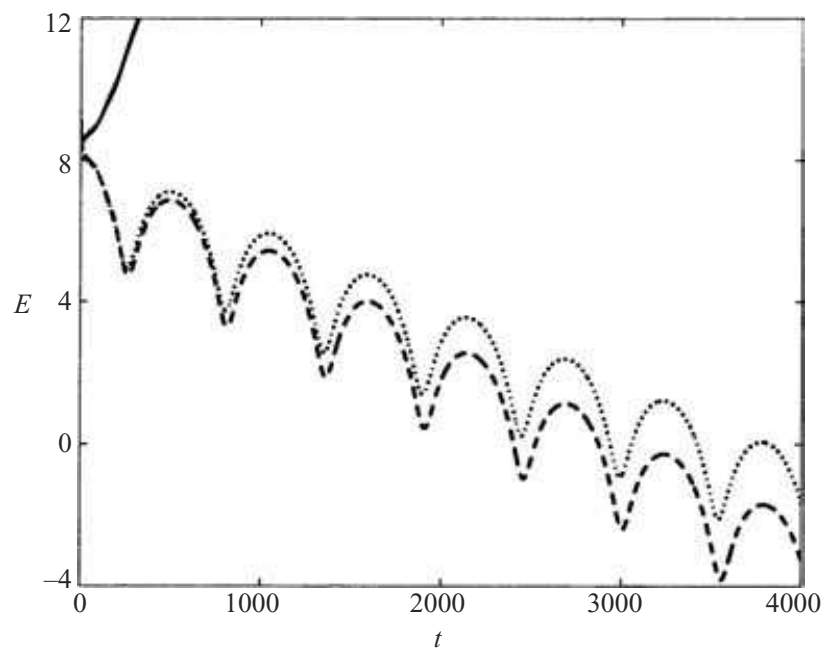

FIGURE 15. Comparison between full-state information control and partial-state information control for $\Omega=\Omega_{B}+1, N=4$ and $\ell=1$ : perturbation energy $E$ as a function of time $t$ on a semi-log plot. Solid line: no control; dashed line: full-state information control; dotted line: partial-state information control with four sensors.

We also introduce the notation $\tilde{\psi}_{i}=\mathscr{C} \psi_{i}(z)$ for $i=1 \ldots N$. The partial state vector is decomposed into

$$
\boldsymbol{Y}=\sum_{i=1}^{N} \alpha_{i} \tilde{\psi}_{i}+R,
$$

where the remainder $R$ is such that $\left[\tilde{\psi}_{i}, R\right]=0$ for all $i=1 \ldots N$. Taking the Cartesian scalar product of (7.10) with $\tilde{\psi}_{j}$ yields

$$
\left[\tilde{\psi}_{j}, \boldsymbol{Y}\right]=\sum_{i=1}^{N} \alpha_{i}\left[\tilde{\psi}_{j}, \tilde{\psi}_{i}\right] .
$$

Following the same steps as those leading from (5.5) to (5.7), one finally obtains

$$
\tilde{\Pi}=\tilde{\mathbf{M}}_{2}^{-1} \tilde{\mathscr{P}}_{2},
$$

where $\tilde{\boldsymbol{M}}_{2}$ is the $N \times N$ matrix of angles $\left[\tilde{\psi}_{j}, \tilde{\psi}_{i}\right]$ and $\tilde{\mathscr{P}}_{2}$ is the $N \times 4$ matrix such that $\tilde{P}_{2} \boldsymbol{Y} \equiv\left[\psi_{j}, \boldsymbol{Y}\right](j=1 \ldots N)$.

The effectiveness of partial information control is illustrated in figure 15. Partialstate information control is seen to be slightly less efficient than its full-state counterpart.

\section{Conclusion}

The main results of the study are as follows: the design of an optimal feedback control scheme on a reduced-order system of the least stable modes has been demonstrated to effectively quench the unstable perturbations leading to vortex breakdown in a finite-length tube, in the linear stage. The feedback is implemented by varying the inlet circulation accordingly. When the reduced order is chosen to be larger than $N=4$, the columnar state is preserved for swirl parameter settings 
$13 \%$ above global instability onset. The control effort approaches zero as time goes to infinity. It should be stressed that the closed-loop control procedure will remain effective for all possible initial conditions since superposition holds as long as the linear approximation is valid. This type of control strategy appears to be promising for experimental implementation: the gain matrix pertaining to the reduced-order system is calculated once and for all off-line. Furthermore, the control law has been shown to be robust with respect to noise, as well as uncertainty in the parameter settings, without resorting to a robust control formulation, as for instance in Bewley \& Liu (1998). The control scheme has also been demonstrated to remain effective even when only partial state information is available. Furthermore, with $N \geqslant 6$, the effective controllability limit can be increased up to $28 \%$ above global instability onset provided the actuator is chosen to be the radial velocity perturbation at the outlet.

It may legitimately be asked whether this control approach could remain effective further away from threshold by increasing the order of the reduced system. Attempts in this direction have not been conclusive: as $N$ is increased, the identification of the modes becomes difficult since their streamwise structure consists of a superposition of Kelvin waves, some of which may be exponentially small with respect to others. This issue may also be related to the method used to project the full system on a reduced-order subspace. Note that instead of using the adjoint eigenmodes as a biorthogonal basis we deliberately chose the original eigenfunctions in the scalar product projections (4.13) and (5.5). This method is valid but it leads to the inversion of ill-conditioned 'matrices of angles' $\boldsymbol{M}$ in the 'receptivity matrix' $\boldsymbol{B}$ (see (4.15)) and $\boldsymbol{M}_{2}$ in the projector $\Pi$ (see (5.7)). One may wonder why the adjoint eigenfunctions were not used instead, as in a classical Galerkin procedure. Such a formulation was indeed attempted: the standard derivation of the adjoint problem to the $(K, \psi)$ original problem (3.2)-(3.3) presented unforeseen difficulties, particularly in that it led to too many adjoint boundary conditions. This inconsistency is most likely related to the $(K, \psi)$ formulation. It does not arise in the primitive variable approach.

The present study raises the following question: what is the reason for the discrepancy between the controllability limit in the reduced-order subspace (equal to the swirl setting where the $(N+1)$ th mode becomes unstable) and the lower effective controllability limit obtained for the full linear state? In other words, why is there a controllability limit at a swirl value lower than expected? According to the mathematical result stated in Lauga \& Bewley (2002), an unstable mode is stabilizable if and only if its entry in the receptivity matrix $\mathscr{B}$ is non-zero. In the case of our full system (4.4), the entries of the receptivity matrix $\mathscr{B}$ corresponding to unstable modes are all non-zero and there should be no controllability limit.

However, Lauga \& Bewley (2002) have also demonstrated that finite numerical accuracy may result in an effective finite controllability limit for a system which is in principle controllable for all values of the bifurcation parameter. In our case, the unstable modes are found to yield among the smallest entries in the receptivity matrix $\mathscr{B}$. This feature together with the necessarily finite numerical accuracy is thought to be responsible for the discrepancy between the controllability limits in the reduced and full system respectively.

It might be argued that the approach taken in the present study is somewhat unorthodox. According to Cortelezzi \& Speyer (1998), a classical mathematically based methodology would consist in first discretizing the full linear system (as in our case) and then deriving a reduced-order model by neglecting poorly controllable states. In the present context, due to the limited efficiency of the actuator, this procedure 
is not applicable since the unstable modes are among the least controllable (see the above discussion on the entries of the receptivity matrix $\mathscr{B}$.)

The approach taken here also differs from many control studies, in that it does not rely on the design of an estimator. Advantage is taken instead of the projection on a reduced-order space. This procedure, which is physically based, has been demonstrated to yield an efficient closed-loop control scheme even in the presence of partial-state information at a few sensor locations.

The closed-loop strategy proposed in the present study may also be compared with open-loop control schemes. For instance, according to the results of Rusak, Wang \& Whiting (1998), a 13\% delay of breakdown onset above the nominal values $\Omega_{1}$ could also be achieved by adding a jet component to the uniform inlet flow with a maximum relative velocity of $15 \%$. The same result could be obtained with a $10 \%$ pipe contraction (Rusak \& Meder 2002). It should be emphasized that in the above open-loop approaches, the control has to be applied for all times, whereas, in the present closed-loop schemes, the control amplitude is allowed to vanish for large times, thereby greatly reducing the control 'cost'.

The control procedure presented in this study is limited to the case of a columnar state with solid body rotation and plug axial flow. For this simple base flow, the radial variations may be conveniently separated out, provided that one restricts attention to the first branch, as discussed in $\S 3.2$. For more realistic base states, this simplification is ruled out. Future extensions should address the optimal control problem, in these less-idealized situations.

Another non-trivial generalization relates to the control of the nonlinear timedependent Squire-Long equations which incorporate vortex breakdown as a possible finite-amplitude state. The ability of our control strategy to dampen finite-size disturbances above $\Omega_{0}$ remains an open issue: it will depend both on the nonlinear characteristics of the bifurcation, i.e. on the size and shape of the basin of attraction of the different states, and on the linear evolution properties such as the intensity of the transients induced by the non-normality of the operator under consideration. As shown by Bewley \& Liu (1998), optimal control theory, on which our reduced-order control strategy is based, is suitable to limit the intensity of the transients, by reducing the non-orthogonality of the controlled eigenmodes.

The authors are very grateful to E. Lauga for enlightening advice on optimal control theory. C. Cossu and P. Schmid are kindly acknowledged for stimulating discussions. The work is supported by the French Ministry of Defense under grant No. 99001004707588.

\section{REFERENCES}

Althaus, W., BrüCker, C. \& Weimer, C. 1996 Breakdown of slender vortices. In Fluid Vortices (ed. S. I. Green), chap. 9, pp. 373-426. Kluwer.

Andersson, P., Berggren, M. \& Henningson, D. 1999 Optimal disturbances and bypass transition in boundary layers. Phys. Fluids 11, 134-150.

Benjamin, T. B. 1962 Theory of the vortex breakdown phenomenon. J. Fluid Mech. 14, 593-629.

Beran, P. 1994 The time-asymptotic behavior of vortex breakdown in tubes. Computers Fluids 23, 913-937.

Beran, P. \& Culick, F. 1992 The role of non-uniqueness in the development of vortex breakdown in tubes. J. Fluid Mech. 242, 491-527.

Bewley, T. 2001 Flow control: New challenges for a new renaissance. Progr. Aerospace Sci. 37, 21-58. 
Bewley, T. \& LiU, S. 1998 Optimal and robust control and estimation of linear paths to transition. J. Fluid Mech. 365, 305-349.

Billant, P., Chomaz, J.-M. \& Huerre, P. 1998 Experimental study of vortex breakdown in swirling jets. J. Fluid Mech. 376, 183-219.

Brown, G. \& Lopez, J. 1990 Axisymmetric vortex breakdown. Part 2: Physical mechanisms. J. Fluid Mech. 221, 553-576.

BRÜCKER, C. 1993 Study of vortex breakdown by Particle Tracking Velocimetry (PTV). Part 2: Spiral type. Exps. Fluids 14, 133-139.

BrüCKer, C. \& Althaus, W. 1992 Study of vortex breakdown by Particle Tracking Velocimetry (PTV). Part 1: Bubble type vortex breakdown. Exps. Fluids 13, 339-349.

BRÜCKer, C. \& Althaus, W. 1995 Study of vortex breakdown by Particle Tracking Velocimetry (PTV). Part 3: Time-dependent structure and development of breakdown modes. Exps. Fluids 18, 174-186.

Chandrasekhar, S. 1961 Hydrodynamic and Hydromagnetic Stability. Clarendon.

Corbett, P. 2000 Non-modal growth in boundary layers, and its optimal control. PhD thesis, Ecole Polytechnique Fédérale de Lausanne, Switzerland.

Cortelezzi, L., Lee, K., Kim, J. \& Speyer, J. 1998 Skin-friction drag reduction via robust reducedorder liner feedback control. Intl J. Comput. Fluid. Dyn. 11, N1-2:19-92,U5,U6,U7.

Cortelezzi, L. \& Speyer, J. 1998 Robust reduced-order controller of laminar boundary layer transitions. Phys. Rev. E 58, 1906-1910.

Darmofal, D. 1996 Comparisons of experimental and numerical results for axisymmetric vortex breakdown in pipes. Computers Fluids 25, 353-371.

Delery, J. 1994 Aspects of vortex breakdown. Prog. Aerospace Sci. 30, 1-59.

ElLe, B. 1958 An investigation at low speed of the flow near the apex of thin delta wings with sharp leading edges. Ministry of Aviation, UK, ARC Tech. Rep. $R \&$ \& 3176, pp. 1-19.

Escudier, M. 1987 Confined vortices in flow machinery. Annu. Rev. Fluid Mech. 19, 27-52.

Escudier, M., Bornstein, J. \& MaXworthy, T. 1982 The dynamics of confined vortices. Proc. R. Soc. Lond. A 382, 335-360.

Faler, J. \& Leibovich, S. 1977 a Disrupted states of vortex flow and vortex breakdown. Phys. Fluids 20, $1385-1400$.

FALer, J. \& Leibovich, S. $1977 b$ An experimental map of the internal structure of a vortex breakdown. J. Fluid Mech. 86, 313-335.

GunZburger, M. 1995 Flow Control. Springer.

Gursul, I., SRinivas, S. \& BatTa, G. 1995 Active control of vortex breakdown over a delta wing. AIAA J. 33, 1743-1745.

Hall, M. 1972 Vortex breakdown. Annu. Rev. Fluid. Mech. 4, 195-218.

Harvey, J. 1962 Some observations of the vortex breakdown phenomenon. J. Fluid Mech. 14, $585-592$.

Högberg, M., Bewley, T. \& Henningson, D. 2003 Linear feedback control and estimation of transition in plane channel flow. J. Fluid Mech. 481, 149-179.

Högberg, M. \& Henningson, D. 2002 Linear optimal control applied to instabilities in spatial boundary layers. J. Fluid Mech. 470, 151-179.

Howard, L. \& GuPTA, A. 1962 On the hydrodynamic and hydromagnetic stability of swirling flows. J. Fluid Mech. 14, 463-476.

Joshi, S., Speyer, J. \& Kim, J. 1997 A systems theory approach to the feedback stabilization of infinitesimal and finite-amplitude disturbances in plane Poiseuille flow. J. Fluid Mech. 332, $157-184$.

Keller, J., Egli, W. \& Exley, W. 1985 Force- and loss-free transitions between flow states. Z. Angew. Math. Phys. 36, 854-889.

KeLvin, LoRd 1880 Vibrations of a columnar vortex. Phil. Mag. 10, 155-168.

Kopecky, R. \& TORRANCE, K. 1973 Initiation and structure of axisymmetric eddies in a rotating stream. Computers Fluids 1, 289-300.

Lambourne, N. C. \& BRYeR, D. W. 1961 The bursting of leading-edge vortices: some observations and discussion of the phenomenon. Aero. Res. Counc. $R \& M$ 3282, pp. 1-35.

LAUB, A. J. 1991 Invariant subspace methods for the numerical solution of Riccati equations. In The Riccati Equation (ed. S. Bittaini, A. J. Laub \& J. C. Willems), pp. 163-196. Springer. 
LaUga, E. \& Bewley, T. 2002 Modern control of linear global instability in a cylinder wake model. Intl J. Heat Fluid Flow 23, 671-677.

Lee, K., Cortelezzi, L., Kim, J. \& Speyer, J. 2001 Application of reduced order controller to turbulent flows for drag reduction. Phys. Fluids 13, 1321-1330.

Leibovich, S. 1978 The structure of vortex breakdown. Annu. Rev. Fluid Mech. 10, 221-246.

LeIbovich, S. 1984 Vortex stability and breakdown: survey and extension. AIAA J. 22, 1192-1206.

LOPEZ, J. 1994 On the bifurcation structure of axisymmetric vortex breakdown in a constricted pipe. Phys. Fluids 6, 3683-3693.

Mitchell, A. \& Delery, J. 2001 Research into vortex breakdown control. Prog. Aerospace Sci. 37, 385-418.

Or, A., Cortelezzi, L. \& Speyer, J. 2001 Robust feedback control of Rayleigh-Bénard convection. J. Fluid Mech. 437, 175-202.

Peckham, D. H. \& Atkinson, S. A. 1957 Preliminary results of low speed wind tunnel tests on a gothic wing of aspect ratio 1.0. Aero. Res. Counc. CP-508, pp. 16-17.

RaYleigh, LoRd 1916 On the dynamics of revolving fluids. Proc. R. Soc. Lond. A 93, 148-154.

RusaK, Z. \& Meder, C. 2002 Near-critical swirling flow in a slightly contracting pipe. AIAA Paper 2002-2984.

Rusak, Z., Wang, S. \& Whiting, C. 1998 Axisymmetric breakdown of a q-vortex in a pipe. AIAA J. 36, 1848-1853.

SARPKAYA, T. 1971 On stationary and travelling vortex breakdown. J. Fluid Mech. 45, 545-559.

SARPKAYA, T. 1974 Effect of adverse pressure gradient on vortex breakdown. AIAA J. 12, 602-607.

Schmid, P. \& Henningson, D. 2001 Stability and Transition in Shear Flows. Springer.

Shtern, V. \& Hussain, F. 1996 Hysteresis in swirling flows. J. Fluid Mech. 309, 1-44.

SQuire, H. B. 1960 Analysis of the vortex breakdown phenomenon. Miszallaneen der Angewandten Mechanik, pp. 306-312. Berlin: Akademie Verlag.

Szeri, A. \& Holmes, P. 1988 Nonlinear stability of axisymmetric swirling flows. Phil. Trans. R. Soc. Lond. A 326, 327-354.

VorobiefF, P. \& Rockwell, D. 1996 Multiple actuator control of vortex breakdown on a pitching delta-wing. AIAA J. 34, 2184-2186.

WANG, S. \& RUSAK, Z. $1996 a$ On the stability of an axisymmetric rotating flow in a pipe. Phys. Fluids 8, 1007-1016.

Wang, S. \& RuSAK, Z. 1996 b On the stability of non-columnar swirling flows. Phys. Fluids 8, $1017-1023$

WANG, S. \& Rusak, Z. 1997 a The dynamics of a swirling flow in a pipe and transition to axisymmetric vortex breakdown. J. Fluid Mech. 340, 177-223.

WANG, S. \& RUSAK, Z. $1997 b$ The effect of slight viscosity on a near-critical swirling flow in a pipe. Phys. Fluids 9, 1914-1927.

WERLÉ, H. 1960 Sur l'eclatement des tourbillons d'apex d'une aile delta aux faibles vitesses. $L a$ Recherche Aéronautique 74, 23-30.

Zhou, K., Doyle, J. C. \& Glover, K. 1995 Robust and Optimal Control. Prentice-Hall. 